\title{
Ppd-1 Remodels Spike Architecture by Regulating Floral Development in wheat
}

\author{
Yangyang Liu $^{1,2^{\dagger}}$, Lili Zhang ${ }^{1 \dagger}$, Michael Melzer ${ }^{3}$, Liping Shen ${ }^{1}$, Zhiwen Sun ${ }^{1,2}$, Ziying Wang ${ }^{1,2}$, Thorsten \\ Schnurbusch ${ }^{3,4^{*}}$, Zifeng Guo $^{1^{*}}$
}

1. Key Laboratory of Plant Molecular Physiology, Institute of Botany, Chinese Academy of Sciences, Beijing 100093, China; 2. University of Chinese Academy of Sciences, Beijing 100049, China; 3. Leibniz Institute of Plant Genetics and Crop Plant Research, OT Gatersleben 06466 Seeland, Germany; 4. Faculty of Natural Sciences III, Institute of Agricultural and Nutritional Sciences, Martin Luther University Halle-Wittenberg, 06120 Halle, Germany

$\dagger$ These authors contributed equally to this work.

*corresponding author e-mail: thor@ipk-gatersleben.de; zifeng guo@163.com

\begin{abstract}
The determination of spike architecture is critical to grain yield in wheat (Triticum aestivum), yet the underlying mechanisms remain largely unknown. Here, we measured 51 traits associated with spike architecture and floral development in 197 wheat accessions with photoperiod sensitive and insensitive alleles. We included five distinct allele combinations at the Photoperiod-1 (Ppd-1) loci. A systematic dissection of all recorded phenotypes revealed connections between floral development, spike architecture and grain yield. Modifying the durations of spikelet primordia initiation did not necessarily affect spikelet number. In addition, $P p d-1$ loci clearly influenced rachis dry weight, pointing to the rachis vascular system as a potential target for higher yield. Ppd-1 displayed opposite effects on the durations of pre and post-anthesis phases. Ppd-1 controlled carpel size, but not anther size. Finally, the photoperiod-insensitive alleles of $P p d-1$ triggered floral degeneration. In parallel, we profiled the spike transcriptome at six stages and four positions in three Ppd-1 genotypes which consists of 234 samples. Integrating phenotypic and expression data suggested that loss of function in Ppd-1 loci delayed floral degeneration by regulating autophagy and extended floret development by regulating genes in different families. Therefore, we concluded that $P p d-1$ remodels spike architecture by regulating floral development in wheat.
\end{abstract}

\section{Introduction}

Wheat (Triticum aestivum L.) is one of the top three crops cultivated worldwide, based on tons produced each year and arable land dedicated to its cultivation. Improving grain yield is a crucial target in wheat breeding, of which grain number is a key factor, itself determined by spike architecture. A spike generally consists of 20-30 spikelets arranged along the main inflorescence stem (the rachis). Each spikelet may produce 8-12 floret primordia (potential grains) in hexaploid wheat (Guo and Schnurbusch, 2015). However, most floret primordia degenerate during the pre-anthesis stage, leaving 0-4 fertile floret per spikelet, drastically reducing potential grain yield. Floral development and abortion during pre-anthesis may be divided into seven distinct stages on the basis of floret morphology: terminal spikelet (TS), white anther (WA), green anther (GA), yellow anther (YA), tipping (TP), heading (HD), and anthesis (AN). The main feature of TS stage is the completion (or termination) of spikelet initiation (Kirby and Appleyard, 1987). At this stage, the last few primordia do not develop into spikelets but instead form the glumes and floret primordia of a terminal spikelet(Kirby and 
Appleyard, 1987). During WA stage, the meristematic dome initiates 7-9 floret primordia within each individual spikelet (1-3 primordia fewer than the maximum number)(Kirby and Appleyard, 1987). The lemmas of florets 1 and 2 at the base of the spikelet completely enclose the anther and ovary(Kirby and Appleyard, 1987). At the GA stage, the meristematic dome completes the initiation of floret primordia, so that after this stage no more floret primordia will be produced (Guo and Schnurbusch, 2015); the glumes almost completely cover all but the tips of the florets. The spike length peaks at the YA stage (Guo et al., 2018); glumes are fully formed, and the lemmas of the first three florets are visible. At the TP stage, the first awn is visible (Zadoks et al., 1974), at HD stage, half of the spike emerges from the last leaf blade (Zadoks et al., 1974). Finally, at the AN stage, yellow anthers appear along the spike (Zadoks et al., 1974).

Spike architecture is a complicated trait, that is determined by multiple factors. Spike fertility index (i.e. the ratio between grain number per spike and weight of spike chaff) is a critical metric of assimilate distribution between grains and spike chaff (e.g. glume, lemma, palea, rachis). Grain number per spikelet along the main inflorescence also influences spike shape. Spikelet density (the ratio between spike length and spikelet number) also indicates spike compactness. Spikelet fertility (the ratio between fertile and total spikelet number) informs on the proportion of aborted spikelets. In addition, grain size traits (thousand-kernel weight-TKW, grain area, grain width, grain length) all contribute to spike architecture.

The response to photoperiod is major agronomic trait that modulates flowering time. In wheat, photoperiod sensitivity is controlled by three Photoperiod-1 ( $P p d-1)$ genes that are located on chromosomes 2A, 2B, and 2D and designated $P p d-A 1, P p d-B 1$, and $P p d-D 1$, respectively (Scarth and Law, 1983; Wilhelm et al., 2009). Ppd-1 genes belong to the PSEUDO-RESPONSE REGULATOR family of circadian clock and photoperiod regulators. Their photoperiod insensitive alleles are designated by the suffix "a" (e.g., Ppd-1a), and alleles conferring a photoperiod sensitivity have the suffix "b" (e.g., Ppd1b) (Mclntosh et al., 2003; Shaw et al., 2013). In wheat breeding, photoperiod insensitivity has been widely introduced in cultivars where early flowering is desirable to avoid stress or to allow crops to flower even under shortday photoperiods (Kato and Yokoyama, 1992; Worland et al., 1998; Shaw et al., 2013). Ppd-1b alleles follow a diurnal expression pattern: they are highly expressed during the light period, whereas their expression falls to very low levels in dark. By contrast, Ppd-1a alleles lack this diurnal pattern, displaying a constant and high expression pattern ( Beales et al., 2007; Wilhelm et al., 2009). Ppd-1a alleles raise the expression of the wheat Flowering Locus 1 (TaFT1) in leaves, from which the FT protein later moves to the shoot apex to induce flowering (Shaw et al., 2012). Ppd-1 was previously reported to similarly play a key role during supernumerary spikelet formation in wheat (Boden et al., 2015).

Here, we first determined the effects of $P p d-1$ loci on spike architecture and developmental traits in a natural population of 197 wheat accessions, then contributions of $P p \mathrm{~d}-1$ alleles to the measured traits using five $P p d-1$ genotypes that vary in their observed flowering time. By quantifying changes in the transcriptome at six stages and four positions along the spike and in three $P p d-1$ genotypes, we demonstrate that $P p d-1$ remodels spike architecture by regulating floral development in wheat.

\section{Results}

\section{Ppd-1 loci affect spike architecture, floret development, and grain yield in 197 wheat accessions}


To determine the effects of the Ppd-D1 locus on spike architecture and floret development, we genotyped the Ppd-D1 gene in a wheat population of 197 wheat accessions and phenotyped them for 51 traits related to spike architecture and development. We then calculated spike architecture traits separately for photoperiod-sensitive (Ppd-D1b) and photoperiod-insensitive ( $P p d-D 1 a)$ allelic variants at the $P p d-D 1$ locus (Table 1).

The allelic status at the $P p d-D 1$ locus significantly affected 37 traits. When normalized to phenotypic values measured for the photoperiod sensitive $P p d-D 1 b$ alleles, variation ranged from 3 to 20\% (when Ppd-D1a alleles have a positive effect on the trait) and from 3 to $17 \%$ (when $P p d-D 1 a$ alleles negatively affected a trait). Photoperiod-insensitive $P p d-D 1 a$ alleles affected

spike compactness by lowering final spike length by $15 \%$, while also decreasing total spikelet number by $13 \%$ and the number of fertile spikelets by $9 \%$. Because of this unequal effect on total and fertile spikelet numbers, $P p d-D 1 a$ alleles resulted in a modest increase in spikelet fertility (the ratio between fertile and total spikelet number) of $4 \%$, although the statistical significance was weak. However, we did not observe a significant difference in spikelet density (the ratio between total spikelet number and spike length). In addition, Ppd$D 1 a$ (the insensitive allele) increased TKW, spike fertility index (that is the ratio between grain number per spike and weight of spike chaff) and the ratio of grain weight/spike chaff by $10 \%$, $10 \%$ and $20 \%$, respectively, compared with $P p d-D 1 b$ (the sensitive allele).

One measure of relative spikelet fertility captures the maximum number of floret primordia in three representative spikelets: the apical (A) and basal (B) florets of the third spikelet from the top or bottom of the spike, respectively, while the central floret (C) sits at the center of the spike. Ppd-D1a insensitive alleles resulted in small drop in the maximum number of floret primordia per spikelet at each $A, C$ and $B$ position (annotated as Max. Floret $A / C / B$ in Table 1): $3 \%$ for $A$ spikes, $5 \%$ for $C$ spikes and $6 \%$ for $B$ spikes, compared with the photoperiod-sensitive allele. Looking at the number of grains produced by the apical spikelet, $P p d-D 1 a$ insensitive alleles increased the grain number per spikelet (grain A) by $14 \%$ and floret survival by $16 \%$ (calculated as the ratio between maximum number of floret primordia and grain number, and labeled survival A in Table 1). Moreover, Ppd-D1a insensitive alleles led to a marked reduction of the loss of floret primordia (expressed as the difference between maximum number of floret primordia and grain number) by $14 \%$ (floret primordia $A$ and $B$ ) and $16 \%$ (floret primordia C). Moreover, Ppd-D1a insensitive alleles reduced spike dry weight (DW) by $5 \%$ and the length of all spike developmental stages by $14-17 \%$. That Ppd-D1a insensitive alleles lowered maximum number of floret primordia, spike length and spike DW may be attributable to their shortening of each spike developmental stage (Table 1).

We also determined grain yield in eight different field environments, as well as spike dry weight in two field environments and grain number in three field environments. Ppd-D1b sensitive alleles consistently increased spike dry weight and grain number, possibly as a consequence of their prolonging spike developmental stages. Since grain yield is a complicated trait that may be influenced by tiller number, grain number, and TKW, Ppd-D1a insensitive alleles had significant positive effects on grain yield in two environments of $\sim 3 \%$ and significant negative effects in three environments of 3-5\%. These results show that allelic variants of $P p d-$ $D 1$ clearly influenced spike architecture and floret developmental traits.

Table 1. Effects of sensitivity and insensitivity alleles at the Ppd-D1 (chromosome 2D) locus on spike architecture and floret development traits.

\begin{tabular}{lllll}
\hline Traits & Sensitivity $(\mathbf{n}=165)$ & Insensitivity $(\mathbf{n}=\mathbf{3 2})$ & Difference (\%) & T-test \\
\hline Final Spike length $(\mathrm{cm})$ & $11.47 \pm 1.29$ & $9.71 \pm 1.07$ & -15 & $1.53 \mathrm{E}-11^{* * *}$
\end{tabular}


bioRxiv preprint doi: https://doi.org/10.1101/2020.05.11.087809; this version posted May 12, 2020. The copyright holder for this preprint (which was not certified by peer review) is the author/funder. All rights reserved. No reuse allowed without permission.

\begin{tabular}{|c|c|c|c|c|}
\hline Total spikelet number & $25.28 \pm 2.87$ & $22.03 \pm 2.1$ & -13 & $6.71 \mathrm{E}-09^{* * *}$ \\
\hline Fertile spikelet number & $22.21 \pm 2.81$ & $20.28 \pm 1.81$ & -9 & $0.0003^{* * *}$ \\
\hline Spikelet fertility & $0.89 \pm 0.09$ & $0.92 \pm 0.04$ & +4 & $0.0248^{*}$ \\
\hline Spike DW (g) & $3.76 \pm 0.68$ & $3.56 \pm 0.4$ & -5 & 0.1115 \\
\hline Grain number per spike & $68.75 \pm 12.58$ & $61.82 \pm 8.63$ & -10 & $0.0092^{* *}$ \\
\hline Grain weight per spike spike & $2.78 \pm 0.6$ & $2.73 \pm 0.31$ & -2 & 0.7177 \\
\hline Spike chaff (g) & $1.31 \pm 0.95$ & $1.43 \pm 1.21$ & +9 & 0.5575 \\
\hline TKW (g) & $40.87 \pm 6.74$ & $44.9 \pm 6.42$ & +10 & $0.0063^{* *}$ \\
\hline Spikelet density & $2.22 \pm 0.24$ & $2.28 \pm 0.21$ & +3 & 0.1664 \\
\hline Spike fertility index & $72.03 \pm 14.5$ & $79 \pm 14.52$ & +10 & $0.0288^{*}$ \\
\hline Grain weight: spike chaff & $2.91 \pm 0.64$ & $3.51 \pm 0.61$ & +20 & $2.60 \mathrm{E}-05^{* * *}$ \\
\hline Max.Floret A & $9.1 \pm 0.55$ & $8.83 \pm 0.48$ & -3 & $0.0151^{*}$ \\
\hline Max.Floret C & $9.87 \pm 0.59$ & $9.34 \pm 0.51$ & -5 & $6.20 \mathrm{E}-06^{* * *}$ \\
\hline Max.Floret B & $9.72 \pm 0.7$ & $9.13 \pm 0.56$ & -6 & $2.15 \mathrm{E}-05^{* * *}$ \\
\hline Grain A & $2.6 \pm 0.77$ & $2.97 \pm 0.52$ & +14 & $0.011^{*}$ \\
\hline Grain C & $3.71 \pm 0.5$ & $3.85 \pm 0.39$ & +4 & 0.127 \\
\hline Grain B & $3.56 \pm 0.6$ & $3.57 \pm 0.45$ & +0.3 & 0.9233 \\
\hline Survival A & $0.29 \pm 0.09$ & $0.33 \pm 0.06$ & +16 & $0.0051^{* *}$ \\
\hline Survival C & $0.38 \pm 0.05$ & $0.41 \pm 0.04$ & +9 & $0.0013^{* *}$ \\
\hline Survival B & $0.37 \pm 0.07$ & $0.39 \pm 0.05$ & +5 & 0.1177 \\
\hline Floret primordia loss A & $6.5 \pm 0.96$ & $5.59 \pm 1.86$ & -14 & $8.48 \mathrm{E}-05^{* * *}$ \\
\hline Floret primordia loss C & $6.15 \pm 0.76$ & $5.19 \pm 1.78$ & -16 & $1.95 \mathrm{E}-06^{* * *}$ \\
\hline Floret primordia loss B & $6.15 \pm 0.93$ & $5.28 \pm 1.85$ & -14 & $0.0001^{* * *}$ \\
\hline TS spike length $(\mathrm{cm})$ & $0.37 \pm 0.07$ & $0.39 \pm 0.08$ & +5 & 0.1861 \\
\hline WA spike length $(\mathrm{cm})$ & $2.08 \pm 0.4$ & $2.28 \pm 0.4$ & +9 & $0.0225^{*}$ \\
\hline GA spike length $(\mathrm{cm})$ & $5.52 \pm 0.91$ & $5.18 \pm 0.77$ & -6 & 0.0591 \\
\hline YA spike length (cm) & $10.49 \pm 1.28$ & $9.32 \pm 1.16$ & -11 & $6.01 \mathrm{E}-06^{* * *}$ \\
\hline TP spike length $(\mathrm{cm})$ & $11.94 \pm 1.28$ & $9.97 \pm 1.03$ & -17 & $1.73 \mathrm{E}-13^{* * *}$ \\
\hline HD spike length $(\mathrm{cm})$ & $12.09 \pm 1.28$ & $10.13 \pm 1.1$ & -16 & $5.21 \mathrm{E}-13^{* * *}$ \\
\hline AN spike length $(\mathrm{cm})$ & $11.99 \pm 1.32$ & $10.1 \pm 1.19$ & -16 & $8.85 \mathrm{E}-12^{* * *}$ \\
\hline TS stage (thermal time ${ }^{\circ} \mathrm{Cd}$ ) & $1035.25 \pm 101.57$ & $888.81 \pm 78.02$ & -14 & $8.25 \mathrm{E}-13^{* * *}$ \\
\hline WA stage (thermal time ${ }^{\circ} \mathrm{Cd}$ ) & $1239.52 \pm 99.38$ & $1057.52 \pm 99.1$ & -15 & $2.10 \mathrm{E}-16^{* * *}$ \\
\hline GA stage (thermal time ${ }^{\circ} \mathrm{Cd}$ ) & $1328.57 \pm 105.4$ & $1130.88 \pm 110.18$ & -15 & $5.78 \mathrm{E}-18^{* * *}$ \\
\hline YA stage (thermal time ${ }^{\circ} \mathrm{Cd}$ ) & $1398.72 \pm 117.69$ & $1187.97 \pm 112.76$ & -15 & $4.34 \mathrm{E}-17^{* * *}$ \\
\hline TP stage (thermal time ${ }^{\circ} \mathrm{Cd}$ ) & $1468.08 \pm 136.22$ & $1235.88 \pm 112.17$ & -16 & $2.19 \mathrm{E}-16^{* * *}$ \\
\hline HD stage (thermal time ${ }^{\circ} \mathrm{Cd}$ ) & $1527.87 \pm 144.92$ & $1282 \pm 109.21$ & -16 & $1.73 \mathrm{E}-16^{* * *}$ \\
\hline AN stage (thermal time ${ }^{\circ} \mathrm{Cd}$ ) & $1645.19 \pm 153.89$ & $1367 \pm 118.79$ & -17 & $1.04 \mathrm{E}-17^{* * *}$ \\
\hline Grain yield (ENV 1, T/ha) & $99.01 \pm 6.23$ & $98.07 \pm 4.31$ & -1 & 0.4138 \\
\hline Grain yield (ENV 2, T/ha) & $106.88 \pm 6.13$ & $101.84 \pm 6.69$ & -5 & $4.61 \mathrm{E}-05^{* * *}$ \\
\hline Grain yield (ENV 3, T/ha) & $92.69 \pm 6.35$ & $95.35 \pm 5.15$ & +3 & $0.0275^{*}$ \\
\hline Grain yield (ENV 4, T/ha) & $100.19 \pm 5.14$ & $99.95 \pm 4.75$ & -0.2 & 0.8038 \\
\hline Grain yield (ENV 5, T/ha) & $91.70 \pm 5.49$ & $94.08 \pm 4.17$ & +3 & $0.0216^{*}$ \\
\hline Grain yield (ENV 6, T/ha) & $104.06 \pm 5.79$ & $103.99 \pm 4.44$ & -0.1 & 0.9537 \\
\hline Grain yield (ENV 7, T/ha) & $79.93 \pm 6.41$ & $76.04 \pm 6.86$ & -5 & $0.0023 * *$ \\
\hline Grain yield (ENV 8, T/ha) & $110.98 \pm 5.96$ & $107.27 \pm 6.38$ & -3 & $0.0018^{* *}$ \\
\hline Spike DW (ENV 3, g) & $32.19 \pm 3.52$ & $31.22 \pm 3.75$ & -3 & 0.1622 \\
\hline Spike DW $($ ENV 8, g) & $33.14 \pm 3.72$ & $32.97 \pm 3.51$ & -1 & 0.8167 \\
\hline Grain number (ENV 1) & $51.23 \pm 7.20$ & $47.54 \pm 8.42$ & -7 & $0.0112^{*}$ \\
\hline Grain number (ENV 3) & $54.87 \pm 6.47$ & $49.60 \pm 7.06$ & -10 & $5.39 \mathrm{E}-05 * * *$ \\
\hline Grain number (ENV 4) & $47.60 \pm 5.27$ & $43.81 \pm 5.18$ & -8 & $2.60 \mathrm{E}-04 * *$ \\
\hline
\end{tabular}

The spike length was measured without the awn. The spike chaff refers to the rachis with empty spikelets after removal of grains. Spike fertility index, spikelet density, and fertility were determined based on spikelet number per $\mathrm{cm}$ of spike length, grain number (per spike) $\mathrm{g}^{-1}$ of spike chaff (per spike) at harvest, and the ratio of the fertile and total spikelet numbers. Infertile spikelets were defined as spikelets that did not set any grain (i.e. were completely empty), Fertile spikelets produced at least one grain. Grain A/Max.Floret A, grain C/Max.Floret C, grain B/Max.Floret B refer to the grain number per spikelet/maximum number of floret primordia per spikelet at apical (the third spikelet from the top of spike), central (the spikelet in the center of spike), basal (the third spikelet from the bottom of spike) spikelet, respectively. Survival/floret primordia loss are the ratios/differences between grain number and maximum number of floret primordia. The data were presented as the mean \pm SD (difference $\%, P$ value for T test). The percentage of differences between NIL/KL and WT were are given relative to the values of sensitive allelesity. ${ }^{*}, * *$, and $* * *$ indicate significance levels of $0.05,0.01$, and 0.001 , respectively. For floret development traits: $n=3$; for the spike architecture traits: $n=6$. 


\section{Validating the effects of Ppd-1 loci on spike architecture and floral development traits in Ppd-1 mutants}

To further validate the effects of Ppd-1 loci on spike architecture and development, we measured the same spike- and spikelet-associated phenotypes in five strains differing at the Ppd-1 loci (see plant materials). The spring wheat variety Paragon carries photoperiodsensitive alleles in all three genomes: $P p d-A 1 b B 1 b D 1 b$, and displays an intermediate flowering time characteristic of this wild type cultivar. Two near-isogenic lines (NILs) have only Ppd-1 insensitive alleles ( $P p d-A 1 a B 1 a D 1 a)$ that result in early flowering. We also characterized two independent mutant lines with loss of function alleles at all three loci ( $p p d-A 1$ B1 D1) that confer late flowering. Although the two NILs obtained their insensitive alleles from different genotypes, they displayed similar spike architecture and floret developmental traits. Similarly, the mutant alleles combined into the two mutant lines originated from different germplasm but showed similar phenotypes. We therefore determined the average phenotypic values for Ppd-A1aB1aD1a and ppd-A1B1D1 by calculating the average of both NILs and both mutant lines.

Ppd-1 also affects floral development and abortion. As expected, Ppd-1 insensitive alleles reduced the time required to complete each of the six stages by $12-17 \%$ when compared to the wild type (WT, carrying only sensitive alleles). By contrast, Ppd-1 loss of function alleles increased the length of each stage by $19-34 \%$ (Table 2). The duration of each stage required for spikelet and floret development and abortion events therefore varied among the three genotypes according to their $P p d-1$ genotypes. The timing of visible floral primordium termination similarly changed as a function of the $P p d-1$ genotype, as shown in Table 2. Indeed, visible floral degradation started during the YA stage and extended into the TP stage in earlyflowering NILs (carrying only insensitive alleles, Ppd-A1aB1aD1a). The abortion of apical florets occurred slightly later in both WT and mutant lines, starting during the TP stage and ending with the HD stage. These results therefore indicated that $P p d-1$ insensitive alleles may trigger floral degeneration.

We observed the maximum number of floret primordia at the GA stage in all three Ppd1 genotypes (Table 2), although the genotype did influence this number as well, as the maximum number of floret primordia was higher in mutant lines compared to NILs and WT. This observation suggests that an extended time window of floral initiation (from the TS to GA stage) in mutant lines increased the number of floret primordia. Although both WT and mutant lines took longer to complete each developmental stage, this did not result in a significant increase in fertile florets per spikelet, as measured at the AN stage, relative to NILs.

Table 2. Numbers of floret primordia at seven stages in WT, NIL and KL.

\begin{tabular}{llllllll}
\hline Genotype & TS & WA & GA & YA & TP & HD & AN \\
\hline NIL & $5.67 \pm 0.47$ & $8.80 \pm 0.40$ & $10.40 \pm 0.49$ & $10.67 \pm 0.75^{1}$ & $5.60 \pm 0.49^{1}$ & $5.57 \pm 0.49$ & $5.33 \pm 0.47$ \\
WT & $6.40 \pm 0.49$ & $8.82 \pm 0.83$ & $10.70 \pm 0.64$ & $10.38 \pm 0.48$ & $9.80 \pm 1.08^{1}$ & $6.00 \pm 0.53^{1}$ & $5.70 \pm 0.46$ \\
KL & $5.00 \pm 0.82$ & $8.91 \pm 0.67$ & $11.63 \pm 0.48$ & $11.29 \pm 0.70$ & $11.00 \pm 0.63^{1}$ & $5.80 \pm 0.40^{1}$ & $5.57 \pm 0.49$
\end{tabular}

The Ppd-1 genotypes for the three lines are Ppd-A1bB1bD1b for wild type (WT), Ppd-A1aB1aD1a for the near-isogenic line (NIL) and $p p d-A 1 B 1 D 1$ for the triple mutant line (KL). Ppd-A1bB1bD1b are $P p d-1$ sensitive (late flowering) alleles in the A, B and D genomes; Ppd-A1aB1aD1a are Ppd-1-insensitive (early flowering) alleles in the A, B and D genomes; and $p p d-A 1, p p d-$ $B 1$ and $p p d-D 1$ represent loss of function alleles of $P p d-1$ in the $A, B$ and $D$ genomes, as the flowering times of plants with these genotypes were later than that of the wild type. The changes in the numbers of floret primordia within individual spikelets were determined across the 7 spike developmental stages: terminal spikelet stage (TS, the stage before the WA stage), WA, GA, YA, TP, HD and AN. At the TS stage, there is no obvious floral structure (e.g., carpel and anther), so we collected samples for transcriptome from tipping stage. Data are presented as the mean $\pm S D, n=3$ for all the stage. ${ }^{1}$ Indicates apical floral abortion. 
Ppd-1 markedly affects ovary width but not anther length. We determined carpel width and anther length during the AN stage in three $P p d-1$ genotypes NILs (early-flowering, $P p d$ $A 1 a B 1 a D 1 a$ ), WT (intermediate flowering, Ppd-A1bB1bD1b), and mutant lines (late flowering, $p p d-A 1 B 1 D 1)$ in the first 4 florets starting from the base of the spike, designated F1 to F4 (Table 3). The mutant lines displayed increased carpel width at all 4 floret positions compared to WT in the range of $9-19 \%$, while the NILs showed reduced carpel width by $12-26 \%$ (Table 3). We hypothesize that the extension of the floret growth period by $67 \%$ (between the TS and AN stages) in the mutant lines relative to WT allowed more time for carpel growth and led to wider carpels. Conversely, the $8 \%$ shorter time for floret growth experienced by the NILS resulted in thinner carpels over the same developmental window. In addition, the genotypes at the $P p d-1$ loci affected the lengths of all six stages equally: photoperiod-insensitive alleles accelerated all stages by $12-18 \%$, and $p p d-1$ loss of function alleles delayed each stage by $27-$ $34 \%$. By contrast, anther length remained relatively stable among the three $P p d-1$ genotypes.

Table 3. Effects of Ppd-1 on floret primordia initiation and development traits.

\begin{tabular}{llll}
\hline Traits & NIL Ppd-A1aB1aD1a (difference \%, T test) & WT Ppd-A1bB1bD1b & KL ppd-A1B1D1 (difference \%, T test) \\
\hline F1 anther length $(\mu \mathrm{m})$ & $3931 \pm 432 \mathrm{a}(-7,0.2637)$ & $4235 \pm 234 \mathrm{a}$ & $4376 \pm 248 \mathrm{a}(+3,0.5024)$ \\
F1 carpel width $(\mu \mathrm{m})$ & $2179 \pm 339 \mathrm{c}\left(-21,0.0309^{*}\right)$ & $2748 \pm 379 \mathrm{~b}$ & $3137 \pm 158 \mathrm{a}(+14,0.0586)$ \\
F2 anther length $(\mu \mathrm{m})$ & $3977 \pm 447 \mathrm{a}(-9,0.1486)$ & $4372 \pm 255 \mathrm{a}$ & $4303 \pm 226 \mathrm{a}(-2,0.7158)$ \\
F2 carpel width $(\mu \mathrm{m})$ & $1854 \pm 197 \mathrm{~b}\left(-26,0.0148^{*}\right)$ & $2490 \pm 418 \mathrm{a}$ & $2770 \pm 358 \mathrm{a}(+11,0.2983)$ \\
F3 anther length $(\mu \mathrm{m})$ & $3720 \pm 291 \mathrm{~b}(-9,0.0895)$ & $4092 \pm 259 \mathrm{ab}$ & $4120 \pm 239 \mathrm{a}(+1,0.8835)$ \\
F3 carpel width $(\mu \mathrm{m})$ & $1655 \pm 73 \mathrm{~b}(-12,0.1491)$ & $1875 \pm 295 \mathrm{ab}$ & $2234 \pm 334 \mathrm{a}(+19,0.1064)$ \\
F4 anther length $(\mu \mathrm{m})$ & $3251 \pm 260 \mathrm{~b}(-10,0.1228)$ & $3610 \pm 308 \mathrm{ab}$ & $3775 \pm 125 \mathrm{a}(+5,0.3994)$ \\
F4 carpel width $(\mu \mathrm{m})$ & $1309 \pm 141 \mathrm{~b}(-14,0.0845)$ & $1519 \pm 175 \mathrm{ab}$ & $1657 \pm 290 \mathrm{a}(+9,0.4027)$ \\
MAX. Floret & $10.67 \pm 0.75 \mathrm{~b}(+3,0.4065)$ & $10.38 \pm 0.48 \mathrm{~b}$ & $11.29 \pm 0.70 \mathrm{a}\left(+9,0.0057^{* *}\right)$ \\
Fertile floret & $5.33 \pm 0.47 \mathrm{a}(-6,0.1736)$ & $5.70 \pm 0.46 \mathrm{a}$ & $5.57 \pm 0.49 \mathrm{a}(-2,0.6124)$ \\
TS stage (days) & $64.25 \pm 0.43 \mathrm{c}\left(-12,1.49 \mathrm{E}-07^{* * *}\right)$ & $73.00 \pm 0.63 \mathrm{~b}$ & $86.67 \pm 0.47 \mathrm{a}\left(+19,1.35 \mathrm{E}-07^{* * *}\right)$ \\
WA stage (days) & $68.25 \pm 0.43 \mathrm{c}\left(-15,4.10 \mathrm{E}-19^{* * *}\right)$ & $80.67 \pm 0.75 \mathrm{~b}$ & $102.22 \pm 1.47 \mathrm{a}\left(+27,1.63 \mathrm{E}-34^{* * *}\right)$ \\
GA stage (days) & $71.82 \pm 0.98 \mathrm{c}\left(-16,1.59 \mathrm{E}-31^{* * *}\right)$ & $86.00 \pm 0.00 \mathrm{~b}$ & $113.33 \pm 2.98 \mathrm{a}\left(+32,4.83 \mathrm{E}-28^{* * *}\right)$ \\
YA stage (days) & $76.16 \pm 1.35 \mathrm{c}\left(-15,3.17 \mathrm{E}-26^{* * *}\right)$ & $90.00 \pm 0.00 \mathrm{~b}$ & $116.44 \pm 1.00 \mathrm{a}\left(+29,1.03 \mathrm{E}-30^{* * *}\right)$ \\
TP stage (days) & $77.41 \pm 1.50 \mathrm{c}\left(-18,1.29 \mathrm{E}-67^{* * *}\right)$ & $94.00 \pm 0.00 \mathrm{~b}$ & $122.00 \pm 0.24 \mathrm{a}\left(+30,7.92 \mathrm{E}-84^{* * *}\right)$ \\
HD stage (days) & $79.54 \pm 1.95 \mathrm{c}\left(-18,7.16 \mathrm{E}-54^{* * *}\right)$ & $97.00 \pm 0.00 \mathrm{~b}$ & $128.31 \pm 0.95 \mathrm{a}\left(+32,7.88 \mathrm{E}-87^{* * *}\right)$ \\
AN stage (days) & $83.50 \pm 1.94 \mathrm{c}\left(-17,3.64 \mathrm{E}-59^{* * *}\right)$ & $101.00 \pm 0.00 \mathrm{~b}$ & $135.63 \pm 0.48 \mathrm{a}\left(+34,2.44 \mathrm{E}-115^{* * *}\right)$ \\
TS-GA (days) & $15.32 \pm 1.51 \mathrm{c}\left(-32,3.41 \mathrm{E}-11^{* * *}\right)$ & $22.67 \pm 1.75 \mathrm{~b}$ & $37.20 \pm 4.21 \mathrm{a}\left(+64,1.71 \mathrm{E}-09^{* * *}\right)$ \\
TS-AN (days) & $25.26 \pm 0.89 \mathrm{c}\left(-8,0.0013^{* *}\right)$ & $27.33 \pm 1.37 \mathrm{~b}$ & $45.58 \pm 4.89 \mathrm{a}\left(+67,4.54 \mathrm{E}-11^{* * *}\right)$ \\
AN-PM (days) & $40.74 \pm 2.58 \mathrm{a}(+6,0.1263)$ & $38.50 \pm 4.84 \mathrm{a}$ & $31.83 \pm 1.07 \mathrm{~b}\left(-17,0.0002^{* * *}\right)$ \\
\hline
\end{tabular}

The data were presented as the mean \pm SD (difference \%, $\mathrm{P}$ value for $\mathrm{T}$ test). For the time to the stages $\mathrm{n}=6$, for the remain traits $\mathrm{n}=3$. The percentage of differences between NIL(Ppd-A1aB1aD1a)/KL ( $p p d-A 1 B 1 D 1)$ and WT were relative to the values of WT. F1, F2, F3, F4 mean the first, second, third, fourth florets from the bottom of the spikelet. The ways of measuring anther length and carpel width were showed in (Guo et al., 2015). MAX. Floret and Fertile floret were determined at GA and AN stages, respectively (Figure $2 \mathrm{~B}$ ). PM, physiological maturity. ${ }^{*},{ }^{* *}$, and ${ }^{* * *}$ suggest significance levels of $0.05,0.01$, and 0.001 , respectively.

\section{Differences of Floral Development Induced by Ppd-1 Loci Explain their Effects on Spike Architecture}

When compared to WT (with photoperiod-sensitive Ppd-1 alleles and intermediate flowering time), the photoperiod-insensitive $P p d-1$ alleles in NILs greatly reduced a number of spikerelated traits: spike length ( $24 \%$ decrease), total spikelet number ( $26 \%$ decrease), and fertile spikelet number (24\% decrease) (Table 4). We hypothesized that the reduction in the duration of spikelet primordia initiation caused by the loss of function ppd-1 alleles carried by the mutant lines (and responsible for late flowering) during the TS stage was responsible for these negative effects (Table 3). However, the clear extension of spikelet primordia production during the TS stage was not accompanied by a significant change in spikelet number. The 
photoperiod-insensitive Ppd-1 alleles in NILs positively influenced spikelet fertility (the ratio between fertile spikelet number and total spikelet number), suggesting that lower total spikelet numbers caused a less fraction of spikelets to abort (Table 4, Figure 1).

Surprisingly, we observed opposite effects on grain number per spikelet and grain number per spike caused by the photoperiod-insensitive Ppd-1 alleles in NILs: they reduced grain number per spike in NILs relative to WT by $24 \%$, while raising grain number per spikelet (relative to WT) by $8 \%$ for grain A, $4 \%$ for grain C, and $6 \%$ for grain B (Table 4 , Figure 1 ). The mutant lines exhibited a significant increase in fertile spikelet number, which may be explained by the opposite effects on grain number/spikelet/spike brought on by ppd-1 loss of function alleles (Table 4, Figure 1). Compared to WT, NILs increased grain weight per spike by $24 \%$. Consistent with the proposed roles of Ppd-1 alleles, mutant lines lowered the same phenotype by $32 \%$.

The ppd-1 alleles in the mutant lines significantly increased spike chaff weight (the dry weight of a spike after removal of grains) by $65 \%$ (Table 4, Figure 1), which may be attributed to their lengthening of floret development stages (TS-AN, $+67 \%$, Table 3 ). All spike chaff components (e.g. glume, lemma, palea, and rachis) complete their differentiation before anthesis (AN stage). The ppd-1 alleles in mutant lines differentially affected the duration of developmental stages pre- and post-anthesis: they extended all phases before anthesis, but shortened other phases that take place after anthesis, for example grain filling (Table 3). A shorter grain filling period (by $17 \%$ between AN and PM, Table 3 ) in the mutants resulted in shrivelled grains (Table 4), which counteracted any previous positive effects measured on carpel size at anthesis (Table 3 ).

To further investigate the effects of $P p d-1$ loci on spike architecture traits (e.g. grain number per spike, grain weight per spike, spike chaff components), we dissected spikes into spikelets and florets to quantify the effects on spikelet and floret organs (e.g. carpel, glume, lemma, palea). Ppd-1a alleles from NILs significantly increased the dry weight of F1, F2, F3, F4 florets, resulting in an overall rise in individual spikelet dry weight by $69 \%$ (Table 4). Similarly, NILs markedly improved individual grain weight for all floret positions: $41 \%$ for floret F1, 47\% for floret F2, 74\% for floret F3 and 264\% for floret F4 (Table 4). Grain weight contributes over $80 \%$ of total floret weight, and will increase with longer grain filling periods, as observed in NILs (Table 3). By contrast, the shorter grain filling phase experienced by the $p p d-1$ mutant lines led to a reduction in individual grain weight (Table 4). We also determined the effects of Ppd-1 alleles on lemma and palea. Compared with WT, ppd-1 mutants accumulated more dry weight in lemmas at all floret positions: F1 (34\%), F2 (49\%), F3 (74\%), and F4 florets (110\%). (Table 4). NILs had the lowest grain number per spike of all Ppd-1 genotype combinations studied here, they also exhibited the heaviest grains per spike, because their high individual grain weight effectively compensated for their low grain number per spike (Table 4). The phenotypic variants of WT, NILs and KL validated the effects of Ppd-1 allelic variants on spike architecture and developmental traits and display the connections between between spike architecture and development. 


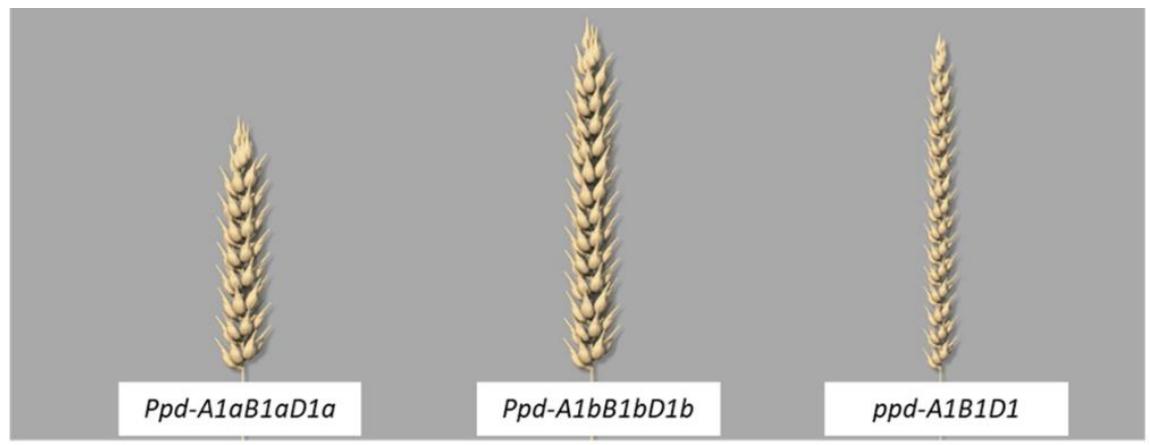

Ppd-A1aB1aD1a (NIL) vs WT:

1. Early flowering,

2. Short spike length

3. Less spikelet number

4. Less grain number per spike

5. Unchanged grain number per spikelet

6. Increased grain weight per spike

7. Unchanged spike chaff
ppd-A1B1D1 (KL) vs WT:

1. Late flowering,

2. Unchanged spike length

3. Unchanged spikelet number

4. Unchanged grain number per spike

5. Less grain number per spikelet

6. Decreased grain weight per spike

7. Increased spike chaff
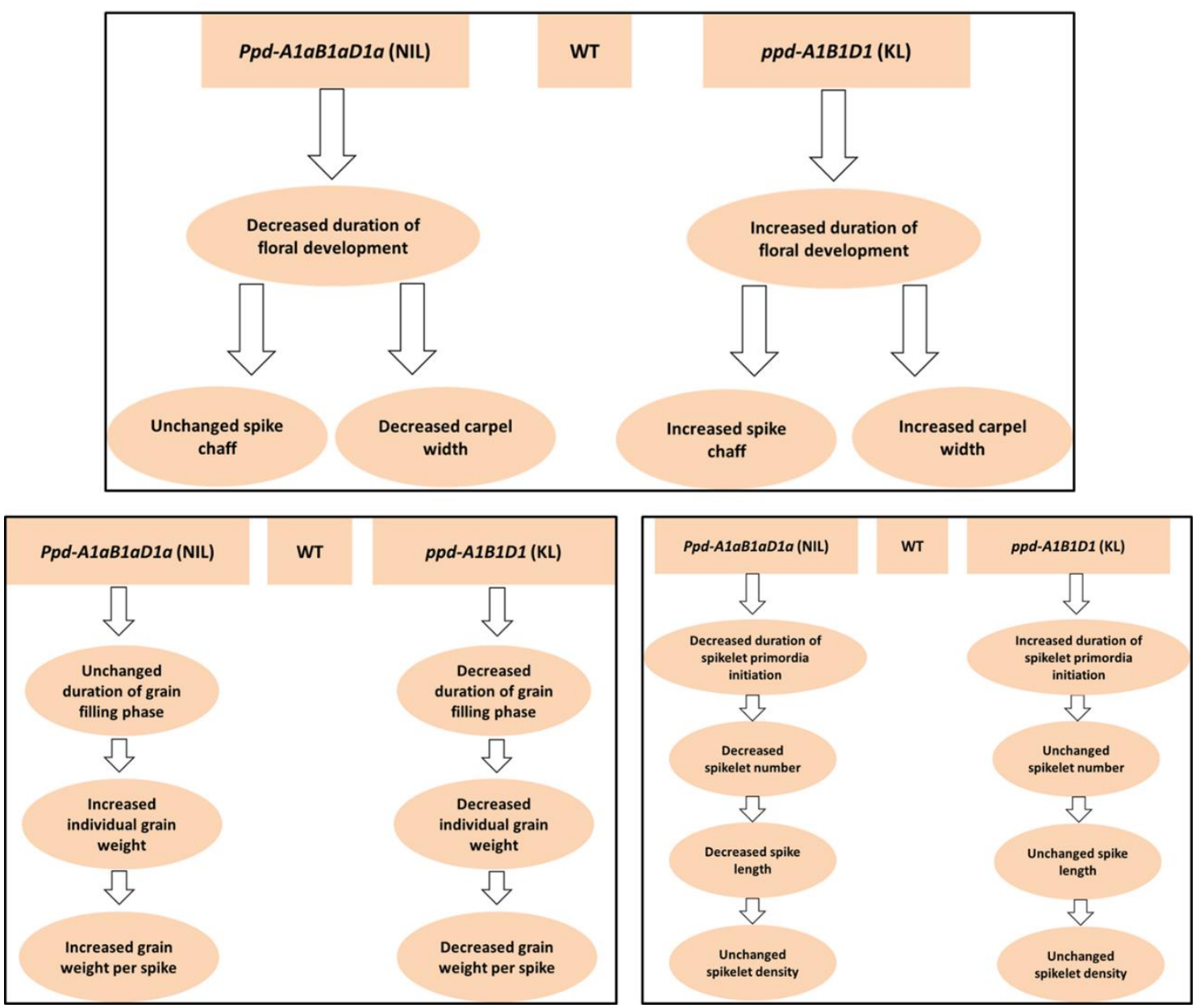

Figure 1. Modification of spike architecture and floral development traits as a function of Ppd-1 genotypes.

Phenotypic differences between NILs (carrying only Ppd-1 insensitive alleles, Ppd-A1aB1aD1a) and mutant lines (loss of function alleles, $p p d-A 1 B 1 D 1)$ compared with WT ( $P p d-1$ sensitive alleles, $P p d-A 1 b B 1 b D 1 b)$ in wheat. Unchanged: no significant difference between $P p d-1$ genotypes. 
Table 4. Effects of Ppd-1 genotypes on spike architecture and floral development traits.

\begin{tabular}{|c|c|c|c|}
\hline Traits & NIL Ppd-A1aB1aD1a (difference \%, T test) & WT Ppd-A1bB1bD1b & $\overline{K L}$ ppd-A1B1D1 (difference \%, T test) \\
\hline Spike length $(\mathrm{cm})$ & $9.34 \pm 0.32 \mathrm{~b}\left(-24,2.19 \mathrm{E}-08^{* * *}\right)$ & $12.24 \pm 0.60 \mathrm{a}$ & $12.84 \pm 0.86 a(+5,0.1528)$ \\
\hline Spike dry weight (g) & $2.50 \pm 0.27 a(+15,0.0948)$ & $2.17 \pm 0.40 \mathrm{a}$ & $2.06 \pm 0.75 a(-5,0.7474)$ \\
\hline Grain weight/spike (g) & $1.97 \pm 0.20 \mathrm{a}\left(+24,0.0224^{*}\right)$ & $1.59 \pm 0.34 a$ & $1.09 \pm 0.66 \mathrm{~b}(-32,0.096)$ \\
\hline Grain number/spike & $54.25 \pm 8.94 b\left(-24,0.0254^{*}\right)$ & $71.00 \pm 15.30 \mathrm{a}$ & $70.75 \pm 12.74 a(-0.35,0.974)$ \\
\hline Total spikelet number & $17.00 \pm 1.00 \mathrm{~b}\left(-26,1.50 \mathrm{E}-06^{* * *}\right)$ & $23.00 \pm 1.73 a$ & $24.75 \pm 2.73 a(+8,0.1738)$ \\
\hline Spikelet density & $1.82 \pm 0.13 a(-3,0.3721)$ & $1.88 \pm 0.09 a$ & $1.94 \pm 0.27 a(+3,0.5903)$ \\
\hline Fertile spikelet number & $16.88 \pm 0.93 b\left(-24,1.67 \mathrm{E}-05^{* *}\right)$ & $22.13 \pm 1.96 a$ & $24.13 \pm 2.67 a(+9,0.1324)$ \\
\hline Spikelet fertility & $0.99 \pm 0.02 a\left(+3,0.0185^{*}\right)$ & $0.96 \pm 0.03 b$ & $0.98 \pm 0.03 \mathrm{ab}(+1,0.3459)$ \\
\hline Spike chaff/spike (g) & $0.45 \pm 0.09 b(-7,0.4365)$ & $0.48 \pm 0.07 \mathrm{~b}$ & $0.79 \pm 0.14 a\left(+65,0.0001^{* * *}\right)$ \\
\hline Spike fertility index & $124.32 \pm 24.33 a(-16,0.0875)$ & $147.86 \pm 23.72 \mathrm{a}$ & $90.33 \pm 13.55 b\left(-39,6.87 \mathrm{E}-05^{* * *}\right)$ \\
\hline Rachis weight (g) & $0.08 \pm 0.02 c\left(-20,0.0462^{*}\right)$ & $0.10 \pm 0.02 b$ & $0.18 \pm 0.04 a\left(+83,0.0001^{* * *}\right)$ \\
\hline Spikelet weight (g) & $0.21 \pm 0.03 a\left(+69,3.55 \mathrm{E}-05^{* * *}\right)$ & $0.12 \pm 0.02 b$ & $0.10 \pm 0.04 b(-17,0.2029)$ \\
\hline Glume weight (g) & $0.0081 \pm 0.0022 \mathrm{a}\left(+36,0.0234^{*}\right)$ & $0.0060 \pm 0.0005 b$ & $0.0096 \pm 0.0011 \mathrm{a}\left(+60,1.57 \mathrm{E}-06^{* * *}\right)$ \\
\hline Grain number $A$ & $3.24 \pm 1.44 a(+8,0.6257)$ & $3.00 \pm 0.45 a$ & $1.55 \pm 1.28 \mathrm{~b}\left(-48,0.0023^{* *}\right)$ \\
\hline Grain number $\mathrm{C}$ & $4.57 \pm 0.58 a(+4,0.443)$ & $4.40 \pm 0.49 a$ & $3.65 \pm 0.96 b\left(-17,0.0333^{*}\right)$ \\
\hline Grain number $B$ & $4.67 \pm 0.56 a(+6,0.2244)$ & $4.40 \pm 0.49 a$ & $1.20 \pm 1.69 \mathrm{~b}\left(-73,4.55 \mathrm{E}-06^{* * *}\right)$ \\
\hline F1 floret weight (g) & $0.0495 \pm 0.0047 a\left(+37,0.0002^{* * *}\right)$ & $0.0361 \pm 0.0055 b$ & $0.0125 \pm 0.00115 \mathrm{c}\left(-65,0.0002^{* * *}\right)$ \\
\hline F1 lemma weight (g) & $0.0041 \pm 0.0014 a b(+14,0.3663)$ & $0.0036 \pm 0.0003 b$ & $0.0048 \pm 0.0005 a\left(+35,4.79 \mathrm{E}-05^{* * *}\right)$ \\
\hline F1 palea weight (g) & $0.0018 \pm 0.0004 a(+15,0.1817)$ & $0.0016 \pm 0.0002 a$ & $0.0017 \pm 0.002 a(+7,0.344)$ \\
\hline F1 grain weight (g) & $0.0438 \pm 0.0041 a\left(+41,0.0002^{* * *}\right)$ & $0.0309 \pm 0.0054 b$ & $0.0058 \pm 0.117 \mathrm{c}\left(-81,0.0001^{* * *}\right)$ \\
\hline F2 floret weight (g) & $0.0524 \pm 0.0050 \mathrm{a}\left(+42,6.79 \mathrm{E}-05^{* * *}\right)$ & $0.0368 \pm 0.0054 b$ & $0.0152 \pm 0.0148 \mathrm{c}\left(-59,0.0028^{* *}\right)$ \\
\hline F2 lemma weight (g) & $0.0041 \pm 0.0008 b\left(+23,0.0333^{*}\right)$ & $0.0033 \pm 0.0003 c$ & $0.0050 \pm 0.0007 a\left(+49,7.44 \mathrm{E}-05^{* * *}\right)$ \\
\hline F2 palea weight (g) & $0.0016 \pm 0.0004 a(+15,0.2153)$ & $0.0014 \pm 0.0002 \mathrm{a}$ & $0.0016 \pm 0.0004 a(+15,0.2343)$ \\
\hline F2 grain weight (g) & $0.0468 \pm 0.0055 a\left(+47,0.0002^{* * *}\right)$ & $0.0320 \pm 0.0053 b$ & $0.0089 \pm 0.0142 c\left(-72,0.0013^{* *}\right)$ \\
\hline F3 floret weight (g) & $0.0498 \pm 0.0055 a\left(+69,5.91 \mathrm{E}-06^{* * *}\right)$ & $0.0295 \pm 0.0052 b$ & $0.0271 \pm 0.0153 b(-8,0.6899)$ \\
\hline F3 lemma weight ( $g$ ) & $0.0032 \pm 0.0008 a\left(+39,0.0119^{*}\right)$ & $0.0023 \pm 0.0003 b$ & $0.0040 \pm 0.0009 a\left(+74,0.0002^{* * *}\right)$ \\
\hline F3 palea weight (g) & $0.00124 \pm 0.0005 b(+5,0.7687)$ & $0.00118 \pm 0.0003 b$ & $0.0016 \pm 0.0003 a\left(+34,0.027^{*}\right)$ \\
\hline F3 grain weight (g) & $0.0453 \pm 0.0059 a\left(+74,1.24 \mathrm{E}-05^{* * *}\right)$ & $0.0260 \pm 0.0050 \mathrm{~b}$ & $0.0216 \pm 0.0148 b(-17,0.462)$ \\
\hline F4 floret weight (g) & $0.0352 \pm 0.0140 \mathrm{a}\left(+184,0.0025^{* *}\right)$ & $0.0124 \pm 0.0087 \mathrm{~b}$ & $0.0227 \pm 0.0144 \mathrm{ab}(+83,0.1379)$ \\
\hline F4 lemma weight (g) & $0.0020 \pm 0.0007 \mathrm{~b}(+32,0.1319)$ & $0.0015 \pm 0.0004 b$ & $0.0032 \pm 0.0008 \mathrm{a}\left(+110,0.0004^{* * *}\right)$ \\
\hline F4 palea weight $(\mathrm{g})$ & $0.0010 \pm 0.0004 a(+39,0.1878)$ & $0.0007 \pm 0.0004 a$ & $0.0011 \pm 0.0006 a(+53,0.1731)$ \\
\hline F4 grain weight (g) & $0.0367 \pm 0.0064 a\left(+264,2.72 \mathrm{E}-05^{* * *}\right)$ & $0.0101 \pm 0.0085 b$ & $0.0184 \pm 0.0140 b(+83,0.209)$ \\
\hline
\end{tabular}

F1, F2, F3, F4 mean the first, second, third, fourth florets from the bottom of the spikelet. Grain A, grain C, grain B mean the grain number per spikelet at apical (the third spikelet from the top of spike), central (the spikelet in the center of spike), basal (the third spikelet from the bottom of spike) spikelet, respectively. The spike length was measured without the awn. The spike chaff refers to the rachis with empty spikelets after removal of grains. Spike fertility index, spikelet density, and fertility were determined based on spikelet number $\mathrm{cm}^{-1}$ of spike length, grain number (per spike) $\mathrm{g}^{-1}$ of spike chaff (per spike) at harvest, and the ratio of the fertile and total spikelet numbers. Infertile spikelets were defined as spikelets that did not set any grain (i.e. were completely empty), whereas fertile spikelets produced at least one grain. The data were presented as the mean $\pm \mathrm{SD}$ (difference \%, $\mathrm{P}$ value for T test). The percentage of differences between NIL/KL and WT were relative to the values of WT. * ${ }^{* *}$, and $* * *$ suggest significance levels of $0.05,0.01$, and 0.001 , respectively. $n=3$ for all the traits.

\section{Spatiotemporal Transcriptome During Loral Development Reveals the Effects of Ppd-1 Loci on Gene Expression}

Spike chaff components (e.g. lemma, glume, palea, rachis) are photosynthetically active and accumulate assimilates during the pre-anthesis phase. Grain number and size are greatly influenced by floret primordia initiation and development during pre-anthesis, and will reflect assimilate partitioning between green tissues and grains. Because spike architecture is closely linked to floret development, a better understanding of the factors that determine spike architecture is critical to discover the mechanisms underlying floret primordia initiation, development and degeneration.

To monitor the development and abortion process of all floret primordia within individual spikelets, we used transcriptome analysis of entire spikelets at six developmental stages (WA, GA, YA, TP, HD, and AN, Figure 2). Since the number of floret primordia peaks 
during the GA stage, and most abort by the AN stage, we collected spikelets starting at the WA stage (preceding the GA stage) to form a baseline before floret primordia numbers reach their maximum. We also collected samples at the YA, TP, and HD stages (before AN) to monitor the progress of floral development and abortion.

To complement our spikelet transcriptome dataset, we also assessed individual florets at three stages (TP, HD, and AN). The basal florets within individual spikelets grow rapidly at these stages, while apical florets are already arrested or aborted. In hexaploid wheat, the first three basal florets (F1, F2, and F3, Figure 2) in spikelets from the central spike always set grains, while the fate of remaining florets (F4-N, Figure 2) is uncertain. We therefore determined the temporal gene expression profile of florets at three stages (TP, HD, and AN) and four positions (F1, F2, F3, and F4-N) within individual spikelets. Since we suspected that $P p d-1$ alleles would play a large role in shaping the transcriptome of spikelets and florets, we obtained samples from the three possible $P p d-1$ genotypes: wild type $P p d-1$ (WT; $P p d-A 1 b B 1 b D 1 b$ ), one NIL (NIL; Ppd-A1aB1aD1a) and one mutant line (KL; ppd-A1B1D1).
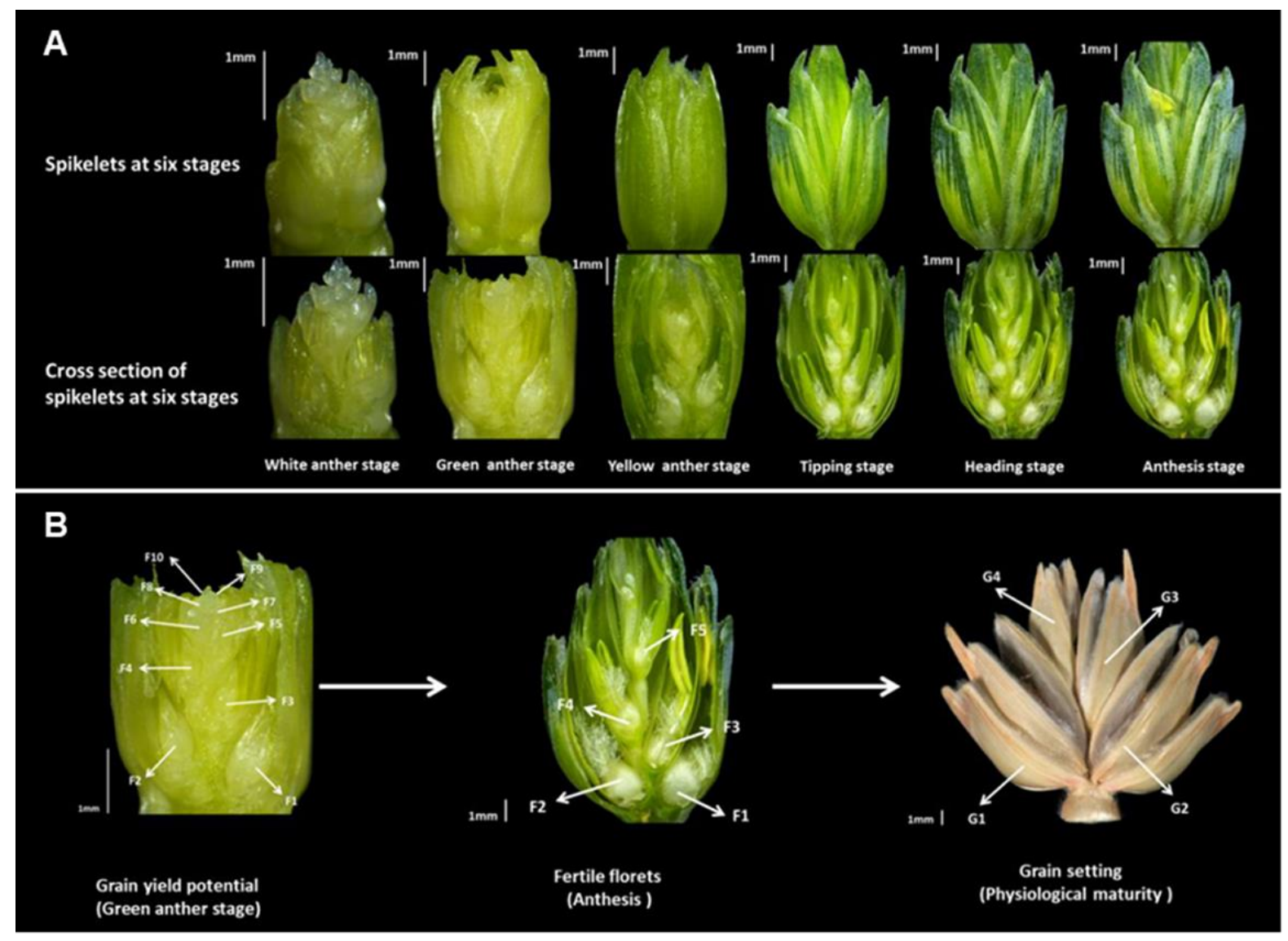

Figure 2. Definition of the developmental stages of wheat spikelets considered in this study. (A) Temporal dissection of spikelet development across six developmental stages. Top row: intact spikelets; bottom row: cross-sections through the central axis. Spikelets start forming floret organs (e.g. anther, carpel, lemma, palea) during the WA stage. Transcriptome data for the entire spikelet (without glumes) were collected at each stage, and transcriptome data for individual florets (florets 1, 2, 3, and 4-N: the floret primordia above the forth floret) were collected at the tipping, heading and anthesis stages.

(B) Spatial dissection of spikelet development. The first three florets (F1, F2 and F3) generally set grains, but florets $\mathrm{F} 4-\mathrm{N}$ do not always, we collected samples for transcriptome analysis 
from all four positions. Florets F1, F2, F3, and F4-N develop into identifiable floret structures (e.g., anther, ovary) at the tipping stage (TS), we therefore started our dissection for transcriptome analysis at this stage. The number of floret primordia peaks at the green anther stage, and reflects grain yield potential. At anthesis, fewer than $50 \%$ of floret primordia have developed into fertile florets. At physiological maturity, fewer than $50 \%$ of floret primordia set grains (G1, G2, G3, and G4). Most floret primordia abort before anthesis.

The number of expressed genes detected in spikelets increased over our developmental time-course, ranging from 37,847 genes during WA to 40,524 during AN (Supplemental Data Set 1). To provide an overview of our expression data set, we generated a heatmap of all differentially-expressed genes (DEGs), which clearly showed waves of distinct gene expression pattern across stages and between genotypes (Figure $3 \mathrm{~A}$ ). We next identified genotype-specific genes involved in spikelet and floret development by comparing our transcriptome datasets across the three $P p d-1$ genotypes. The vast majority (85.1\%) of DEGs displayed no preference for a Ppd-1 genotype across all developmental stages. Genotypespecific genes related to floret development were by contrast relatively rare, accounting for only 1.9 to $3.6 \%$ of all DEGs (Figure 3B, Supplemental Data Set 1). Focusing on floret development, the three $P p d-1$ genotypes largely expressed the same gene sets, with an overlap of $82.8 \%$. Again, genotype-specific differences in gene expression was rather minor, accounting only for 1.65 to $4 \%$ of DEGs (Supplemental Data Set 1 ).

During spikelet development, 9,399 genes were upregulated, against 8,012 downregulated genes over the course of the six stages (Figure $3 \mathrm{C}$ ). When sorted by genotype, 3,593 genes were upregulated for all the six stages in the NIL when compared to WT. The ppd1 mutant line showed even more upregulated genes, with 5,806 genes relative to WT (Figure $3 C)$. A comparable number of genes was downregulated relative to WT: 3,762 genes in the NIL and 4,250 genes in the mutant line (Figure 3C).

We then extracted stage-specific genes from our transcriptome across the six stages (WA, GA, YA, TP, HD, AN). Most genes expressed in spikelets overlapped with those expressed during each of the six stages, accounting for $87.9 \%$ of detected genes (40524 genes). Genes expressed during a single stage of spikelet development made up a much smaller fraction of expressed genes, with 1,992 genes only detected during WA and 1,745 genes specifically expressed during AN (the earliest and latest stages, respectively). Other developmental stages saw even smaller number of stage-specific genes: 129 genes during GA, 227 genes during YA, 296 genes during TP and 507 genes during HD (Figure 3D).

Finally, we investigated genes specifically expressed in florets across all three Ppd-1 genotypes, three stages (TP, HD, and AN) and all four floret positions. Outside of genes whose expression was detected in all conditions, genotype-, stage- and position-specific genes comprised only $1.65-4 \%, 0.9-11.5 \%$ and $0.6-3.4 \%$ of all detected genes, respectively (Supplemental Data Set 1). When focusing on each genotype, we detected 1,568 genes that were expressed only in the NIL (Ppd-A1a B1a D1a), 977 in WT (Ppd-A1b B1b D1b) and 641 in the mutant line ( $p p d-A 1 B 1 D 1)$. 
A

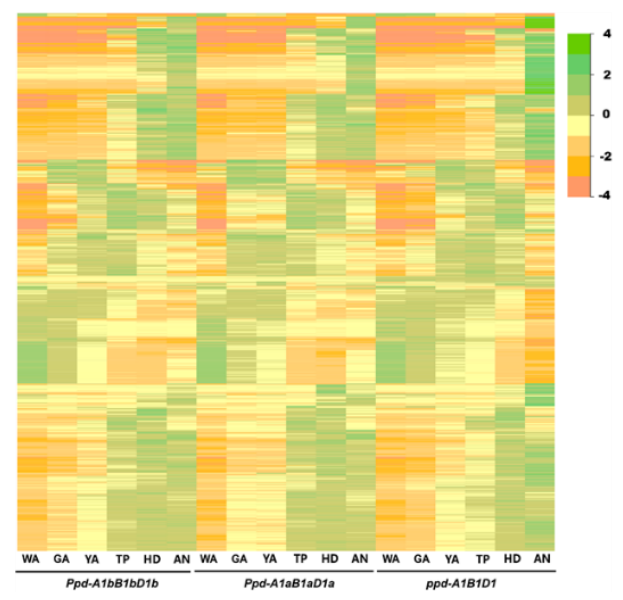

C

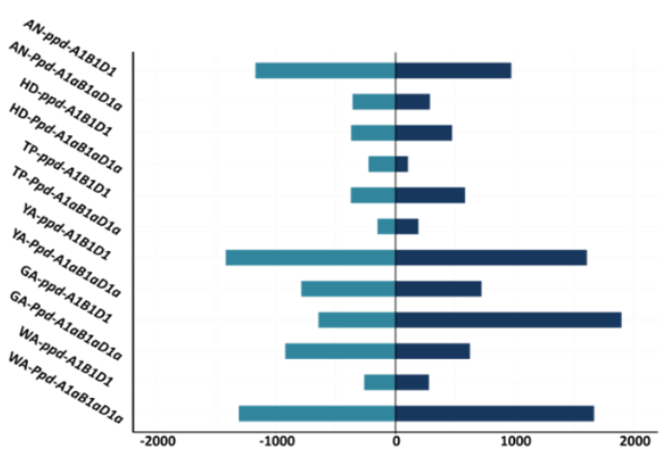

B

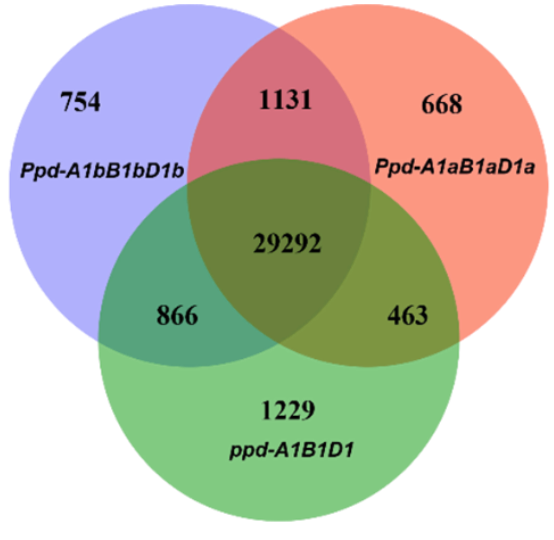

D

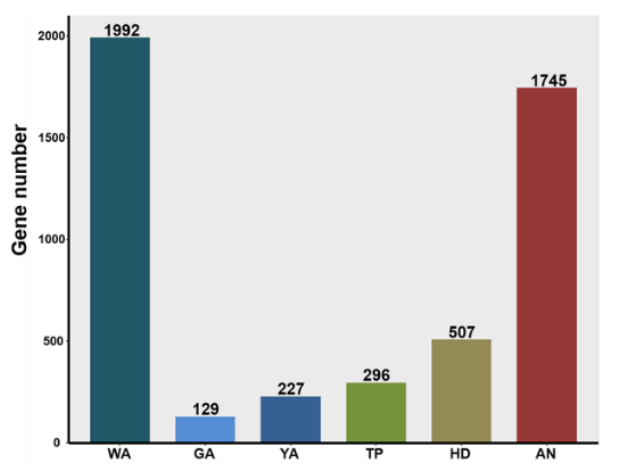

Figure 3. Expression profiles of spikelets across six developmental stages and three genotypes.

(A) Heatmap of differentially expressed genes during spikelet development over the six developmental stages (WA, GA, YA, TP, HD, and AN) and in three genotypes (WT, NILs and mutant lines). Colors indicate relative expression (red, low expression; green, high expression).

(B) Venn diagram of genes expressed in spikelets in each genotype.

(C) Up- and down-regulated genes detected in NILs and mutant lines relative to WT.

(D) Numbers of uniquely expressed genes specific for a single spikelet developmental stage. Four or five biological replicates were collected for each time point. Each replicate was a pool from at least three to ten plants.

\section{Spatiotemporal Changes in Functional Categories during Developmental Transition}

To explore how the transcriptome landscape shapes spikelet and floret transitions during development, we investigated gene ontogeny (GO) terms over the course of our six development stages (Figure 2 ) and in the three $P p d-1$ genotypes. GO-term enrichment analysis during spikelet development revealed that terms such as "carbohydrate catabolic process", "glucose metabolic process" and "response to hormone stimulus" were enriched early during spikelet development in all three genotypes (Figure 4A). The category "response to hormone stimulus" followed a similar spatial pattern during floret development within individual spikelets, as related GO terms were enriched only in basal florets within individual spikelets in all three genotypes (Supplemental Figure 1). These results corroborated previously published data demonstrating the critical importance of hormones and glucose in plant flower development (Song et al., 2018; Figueiredo et al., 2015; Béziat et al., 2017; Moore et al., 2003). These results also validate our own previous work that suggested that hormone- and sugarrelated genes, such as the wheat homologues of SUGAR SIGNALLING IN BARLEY 2, 
BRASSINOSTEROID INSENSITIVE 1 and SUCROSE SYNTHASE 1, were involved in floret development (Guo et al., 2017a).

Conversely, terms such as "cell wall macromolecule metabolic process" and "cell wall polysaccharide metabolic process" were enriched at later spikelet developmental stages in all three genotypes. In support, we observed clear cellular modifications of apical florets as a function of their developmental stage (Figure 4B-4P). While no modifications can be seen during the WA stage, early in spikelet development (Figure 4G, 4L), the initiation of cellular modifications of apical florets is characterized by an increase in the size and number of vacuoles during the GA stage (Figure $4 \mathrm{H}, 4 \mathrm{M}$ ), followed by an increase in vesicle number at the YA stage (Figure $4 \mathrm{I}, 4 \mathrm{~N}$ ). Lipid droplets (L) become visible starting at the TP stage (Figure 4O). Cellular modifications occurred rapidly, starting at the GA stage, and almost all structures were lost by the HD stage: vesicles expanded to engulf much of the cell, lipid droplets took up a large fraction of cellular space, and the initial cell wall structure was lost (Figure 4J-4K, 4O4P). Moreover, visible floral degeneration occurred after the GA stage, most obviously between the YA and HD stages (Figure 4D-4F; Table 2). Consistent with this observation, the GO terms "cellular homeostasis" and "cellular redox homeostasis" were significantly enriched among genes expressed at the YA, TP, and HD stages in all three Ppd-1 genotypes. In this context, we observed more lipid droplets at the TP and HD stage (Figure 4P). However, we did not detect significant enrichment for GO term like "lipid biosynthetic" or "lipid metabolic process" at any of the stages we studied (Figure 4A, Supplemental Figure 1), which calls for further investigation in the future.

Autophagy may be involved in floral abortion in wheat (Ghiglione et al., 2008) and is closely associated with plant lipid homeostasis (Avin-Wittenberg et al., 2015; Fan et al., 2019). AUTOPHAGY-RELATED PROTEIN 8 (ATG8) plays a central role in the autophagy process (Behrends et al., 2010). We therefore determined the expression level of the wheat ATG8 genes from the $A$ and $B$ genomes ( $D$ genome copy not expressed) to explore their potential role in spikelet development. Transcript levels of TaATG-8bA increased starting at the WA stage, while TaATG-B8b displayed the opposite pattern (Figure 4Q, 4R, 4S, Supplemental Data Set 2). In WT, within individual spikelet, TaATG-A8b expression decreased from basal (F1) to apical floret (F4) at TP and HD stages, but displayed an increased trend at AN stage (Supplemental Data Set 3). TaATG-B8b expression increased from basal (F1) to apical floret (F4), but displayed a decreased trend at AN stage (Supplemental Data Set 3). The ubiquitinlike autophagy-related protein ATG12 is required for the early steps of autophagy (Murrow et al., 2015). The expression of TaATG-A12 was much higher than TaATG-B12, and was highly expressed at early developmental stages (WA, GA), concomitant with the first signs of floret primordia degeneration (Supplemental Data Set 2). Within individual spikelets, TaATG-A12 expression increased from the basal floret (F1) to the apical floret (F4), which is consistent with the observed floret primordia phenotype: apical floret primordia are less advanced in their development at this stage, and will generally abort. $\mathrm{KL}$ (ppd-A1B1D1) decreased the expression of TaATG-B8b and TaATG-A12 for spikelet at AN stage (Supplemental Data Set 2). Also, KL (ppd-A1B1D1) decreased the expression of TaATG-B8b for F1, F2, F3, F4 floret at TP stage (Supplemental Data Set 3). The expression of $P p d-1$ is very low in spikelet. Overall, our results indicate that $P p d-1$ loci may regulate floret abortion through their effects on the regulation of autophagy. 

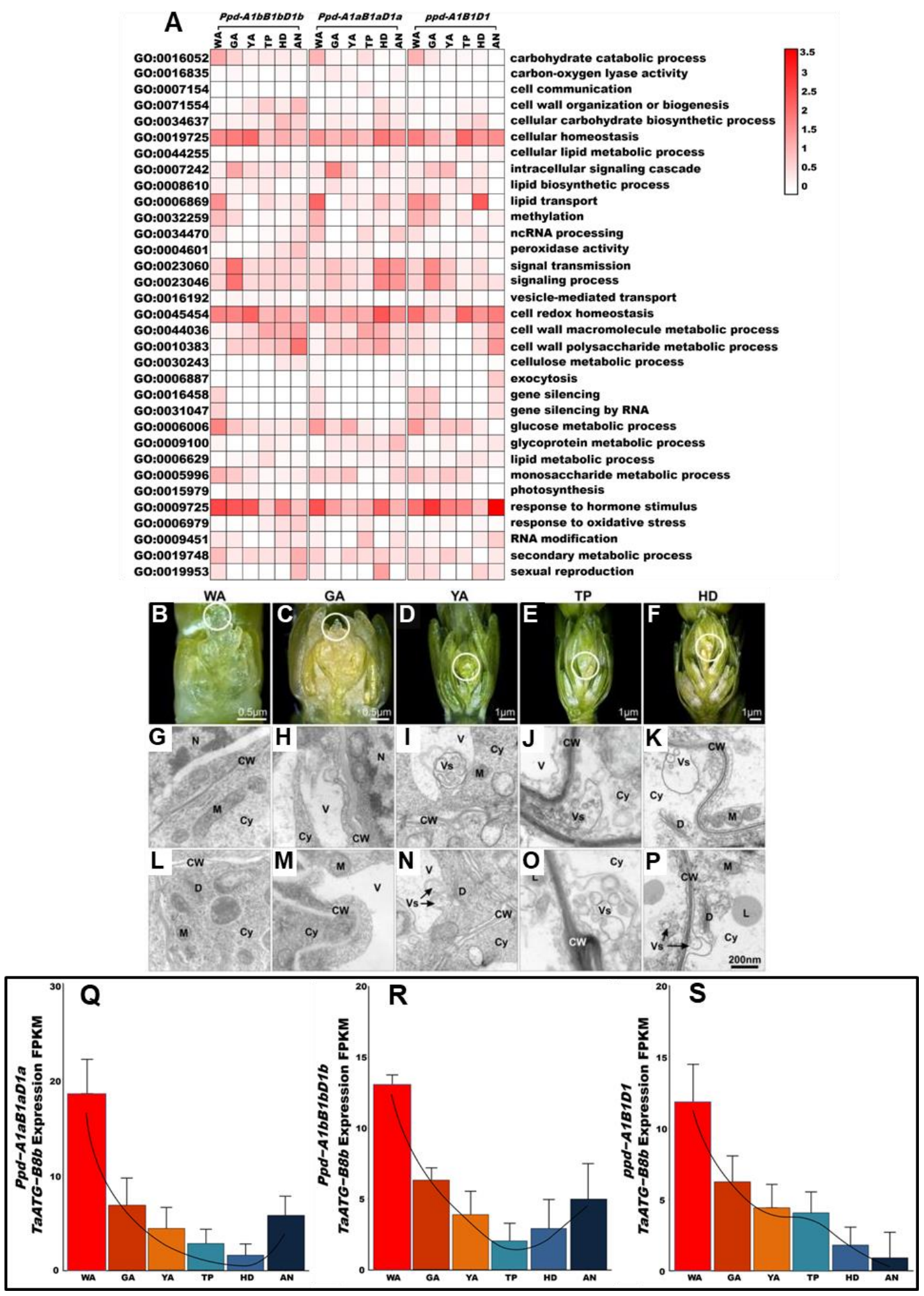

Figure 4. Temporal functional transition of spikelet gene and cellular morphology.

(A) Enrichment in functional categories for genes expressed during spikelet development over six developmental stages in each of the three $P p d-1$ genotypes. The six stages (WA, GA, YA, $T P, H D$, and AN) are as defined in Figure 2. Red indicates dynamic changes across spikelet stages.

(B-P) Modification of cellular morphology across six developmental stages. The paired figures (G-L, H-M, I-N, J-O, K-P) show the different positions of cell at the same stages. The 
transmission electron micrographs reveal the cellular morphology of the part indicated by the white circle. V, vacuole; Cy, cytoplasm; CW, cell wall; L, lipid drops; M, mitochondrion; $\mathbf{D}$, dictyosome; Vs, vesicle.

(Q-S) Expression levels of TaATG-B8b in the three Ppd-1 genotypes (Ppd-A1b B1b D1b, Ppd$A 1 a$ B1a D1a, and ppd-A1 B1 D1) from our transcriptome datasets.

\section{Expression of PIFs MADS-box, SPL, EPFL and HD-ZIP Family Members}

The transition to flowering is controlled by complex genetic networks. Members of multiple gene families, such as MADS-box, SQUAMOSA PROMOTOR BINDING PROTEIN-LIKE (SPL), EPIDERMAL PATTERNING FACTOR-LIKE (EPFL), PHYTOCHROME INTERACTING FACTORS (PIFS) and Homeodomain leucine zipper protein (HD-ZIP) transcription factors, play diverse roles in plant growth and development, including the guidance of inflorescence architecture and floral development. We therefore assessed transcript abundance for all 4 gene families in our transcriptome datasets. We detected 173 DEGs including genes in these four gene families and other genes related to spike development.

PHYTOCHROME INTERACTING FACTOR (PIF) Family PHYTOCHROME INTERACTING FACTORS (PIFs) are basic helix-loop-helix transcription factors that interact physically with the red and far-red light photoreceptors phytochromes (Pham et al., 2018). Arabidopsis PIF1 is unique as it is the only PIF that exclusively represses seed germination in the dark (Oh et al., 2004). The pif1 mutant also displays an early flowering phenotype (Wu et al., 2018). The wheat PIF1 homologue TaPIF-D1 is one of the genes with highest expression (Figure 5A). The expression of TaPIF-D1 decreased from WA to AN stages, but also responded to the status at the $P p d-1$ loci, as its expression decreased with later flowering times. The $p p d-1$ mutant lines significantly lowered the expression of TaPIF-D1 at the AN stage.

MADS-box Family MADS-box genes specify floral organ identity (e.g. sepals, petals, stamens, and carpels). Members of the MADS-box transcription factor family have been studied in a wide range of species (Krizek and Fletcher, 2005), and their role in flower development is well understood at the molecular level. SEPALLATA1 (also known as AGAMOUS-LIKE2, AGL2), SEP2 (AGL4), AGL6, AGL8 and AGL14 are MADS-box genes with sequence similarity to the homeotic gene AGAMOUS. AGL8 expression is not detected in flower primordia and accumulates in the developing carpels in Arabidopsis (Mandel and Yanofsky, 1995). AGL8 also regulates the expression of genes required for cellular differentiation during flower development in Arabidopsis (Gu et al., 1998). We found that the expression of TaAGL-B8 in wheat spikelets reached its highest expression level at the GA stage (446 FPKM) and then decreased during the HD and AN stages (100-200 FPKM), suggesting that TaAGL-B8 may participate in carpel formation during the WA stage (Figure 5B, Supplemental Data Set 4). ppd-1 mutant alleles significantly raised the expression of TaAGL$B 8$ during the WA, GA, and YA stages, but later caused a drop in its expression at the AN stage (Supplemental Data Set 4).. Consistently, within spikelet, TaAGL-B8 displays higher expression at apical florets (F4), which is less advanced florets (Supplemental Data Set 5). It suggests the important role of TaAGL-B8 at early floral development. Ppd-1 knockout significantly increases the expression of TaAGL-B8 for spikelet at WA, GA and YA stages, but decrease its expression at AN stage.

In Arabidopsis, the AGL2 transcript level remains high and constant throughout the floral meristem and in the primordia of all four floral organs: sepals, petals, stamens and carpels (Flanagan and Ma, 1994). We observed here that expression of TaAGL-B2/TaSEP-B1 increased during wheat spikelet development, with lowest levels during the WA stage and reaching peak levels at the $A N$ stage in all the three $P p d-1$ genotypes (Figure $5 C$, Supplemental 
Data Set 4). Consistently, we observed the higher expression of TaAGL-A2 at apical florets (F4, less advanced floret) (Supplemental Data Set 5). It suggests the important role of TaAGL2 at early stage, since the primordia are differentiating into floral organ of sepals, petals, stamens and carpels at early stage. Ppd-1 knockout significantly increases the expression of TaAGL-A2 for spikelet at AN stage (Figure 5D).

AGL4 is also known as SEP2. The transcription factor FAR-RED ELONGATED HYPOCOTYL3 (FHY3) activates AGL4/SEP2 expression to promote floral meristem determinacy (Li et al., 2016). By contrast, TaAGL-B4 expression peaked at the TP stage (Figure 5C), which is consistent with the fast growth of floret/spikelet from between the TP and AN stages. A strong increase in TaAGL-B4 expression in ppd-1 mutant lines was observed at the TP stage.

AGL6 expression is most abundant in developing flowers and ovules (Ma et al., 1991; Schauer et al., 2009). Loss of function of the rice and maize AGL6 homologues affects floral differentiation (Ohmori et al., 2009; Thompson et al., 2009). The APETALA2-like gene AP2L2 plays a critical role in specifying axillary floral meristems and lemma identity (Debernardi et al., 2019). TaAGL-D17 and TaAP2L-B2 expression peaked during the GA and YA stages (the key phase of floret primordia developing into organs), suggesting their important roles in floret differentiation (Figure 5C, Supplemental Data Set 4). The peak in TaAGL-D17 and TaAP2L-B2 expression differed depending on the status of the Ppd-1 loci: WT showed a peak during the GA stage, whereas both NILs (with only the insensitive alleles Ppd-A1a B1a D1a) and the mutant lines (with complete loss of function at all three loci lines: ppd-A1 B1 D1) delay their peaks to the YA stage. Within individual spikelet, we detected higher gene expression of at basal florets (more advanced florets).

XAANTAL2 (XAL2/AGL14) is critical for floral meristem maintenance and determinacy (Pérez-Ruiz et al., 2015). We observed a decrease in the expression of TaAGL-A14 and TaAGL$B 14$, while the expression of $T a A G L-D 14$ increased, suggesting that each gene may carry out distinct functions during spikelet developmental (Figure 5C, Supplemental Data Set 4). Indeed, they reached peak expression levels at different stages: WA stage for TaAGL-A14 and TaAGL$B 14$, and HD stage for TaAGL-D14, regardless of the genotype at the Ppd-1 loci.

Wheat PISTILLATA1 (WPI1) and WPI2 encode the wheat homologues of the Arabidopsis PISTILLATA (PI) MADS-box gene. WPI2 is the probable orthologue of the rice gene OsMADS2 (Hama et al., 2004). The WPI1 gene is expressed in the primordia of lodicules and stamens, but is not detected in the primordia of pistil-like stamens. The suppression of OSMADS2 by RNA interference causes the flowers to fail to open and the transformation of lodicules into palea-like organs (Yadav et al., 2007; Prasad and Vijayraghavan, 2003). From the WA to the AN stage, WPI-A1 globally decreased in expression (Supplemental Data Set 4), while WPI-B2 followed the opposite pattern (Supplemental Data Set 4), indicating that WPI-A1 and $W P I-B 2$ may play different roles during floret/spikelet development. Within individual spikelet, $W P I-A 1$ and $W P I-B 2$ are highly expressed at all the four floret positions. ppd-1 mutant alleles significantly decreased the expression of WPI-B2 during the HD and AN stages.

Previous work found that ful 3 mutants showed delayed flowering time and reduced stem elongation, arguing that FUL3 plays a critical role in spikelet development (Li et al., 2019). Wheat TaMADS28 is the homologue of rice OSMADS31, which is likely involved in salt tolerance during rice seed germination (Yu et al., 2018). We observed an increase in the expression of FUL-D3 and TaMADS-D28 from WA stage, peak expression at YA/TP stage (around 40 FPKM) and a later decrease from YA/TP to AN (Figure 5D, Supplemental Data Set 4). These results suggest that FUL-D3 and TaMADS-D28 may play a role at YA/TP stage, which corresponds to the spike booting phase in wheat (Guo et al., 2018). ppd-1 mutant lines showed a delay in the peak of TaMADS-D28 expression from the YA to the TP stage. Interestingly, we 
detected very low expression of TaMADS-D28 at basal florets and much higher expression at apical florets. Ppd-1 knockout delays the peak of TaMADS-D28 expression from YA to TP stage and decreases its expression at apical florets. FUL-D3 is highly expressed at apical florets (F3, F4) (Supplemental Data Set 5).

FLOWERING LOCUS T (FT) encodes a small globular protein that moves from the leaves to the shoot apex through the phloem (Wigge, 2011). At the shoot apical meristem (SAM), FT interacts with FD to promote the transition of the vegetative meristem into a reproductive inflorescence meristem (Wigge et al., 2005; Collani et al., 2019). In this study, TaFT-B11 and TaFT-D9 showed a consistent increasing trend from WA to AN stage in the wheat spike, and high expression in all the florets (Supplemental Data Set 4). TaFT-B11 and TaFT-D9 show a gradual increase in expression from the WA to the AN stage (Supplemental Data Set 4), suggesting a role during late floret/spikelet development. ppd-1 mutant alleles significantly increased the peak of TaFT-B11 expression, while decreasing the peak of TaFT-D9 expression.

The MADS-box transcription factor FUL and SUPPRESSOR OF OVEREXPRESSION OF CONSTANS 1 (SOC1) redundantly promote flowering (Balanzà et al., 2014) by repressing the expression of SHORT VEGETATIVE PHASE (SVP). During early flower development, APETALA1 (AP1) represses the expression of two redundant flowering time genes, SOC1 and SVP, to prevent floral reversion. During late flower development, such repression is necessary to activate SEPALATA3 (SEP3) (Mutasa-Göttgens and Hedden, 2009). In our transcriptome dataset, TaSOC-D1 expression decreased between the WA and TP/HD/AN stages (Supplemental Data Set 4), which would suggest a key role in early floral development. By contrast, TaSVP-61 expression peaked later (during the GA/YA/TP/HD stages), while TaSVP$D 1$ expression increased from WA to AN (Supplemental Data Set 4), indicating their variable roles in floral development. Also, we observed relatively high expression of TaSOC-D1 at apical florets (F3, F4, less advanced florets) (Supplemental Data Set 5). TaSVP-61 expression peaked during the GA stage in NILs (with photoperiod-insensitive $P p d-1$ alleles), while WT and mutant lines both delayed maximum expression to the TP stage.

In wheat, VERNALIZATION1 (VRN1) regulates the transition from the vegetative to the reproductive phase, as well as the switch between winter and spring growth habits (Yan et al., 2003). The MADS-box genes VRN1, FUL2 and FUL3 all play critical and redundant roles in in the fate of the upper spikelet ridge and the suppression of the lower leaf ridge (Li et al., 2019). VRN1 expression peaked at the YA/TP stage and dropped to its lowest levels during the AN stage (Supplemental Data Set 4), suggesting a specific role at the YA/TP stage. For individual floret, the highest expression of VRN-B1 was observed at apical florets (Supplemental Data Set 5). ppd-1 mutant alleles delayed the peak of VRN1 expression from YA to TP stage. Also, it decreases the expression at apical florets.

SQUAMOSA PROMOTOR BINDING PROTEIN-LIKE (SPL) Family The decision to flower integrates multiple signals, one of them being plant age. This age-dependent pathway is controlled by the microRNA (miR) miR156 and its target genes SQUAMOSA PROMOTER BINDING-LIKES (SPLS) (Cheng, 2004). SPLs are a key hub transcription factors for several flowering pathways in Arabidopsis (Hong and Jackson, 2015). It is unknown whether SPLS regulate flowering in wheat, or how. SPL3 can mediate the activation of APETALA1, LEAFY, and FRUITFULL by the FT-FD complex to induce flowering under inductive long day photoperiods in Arabidopsis (Jung et al., 2016; Yamaguchi et al., 2009). SEPALLATA3 (SEP3) expression was affected in response to altered SPL3 and FT expression in the leaf and shoot apical regions in the regulation of flowering in Arabidopsis in response to ambient temperatures (Lee et al., 2012). SBP transcription factors therefore play critical and varied roles in plant development. As in Arabidopsis, SPL3 and SPL17 were putative targets for tae-miR156 (Xia et al., 2014). We 
observed a pronounced drop in TaSPL-A3 expression from WA/GA/YA to HD/AN stages (Supplemental Data Set 4), while TaSPL-B17 expression decreased from WA to TP/HD/AN stages (Supplemental Data Set 4). ppd-1 loss of function alleles strongly lowered the expression of both TaSPL-A3 and TaSPL-B17 at the AN stage.

HOMEODOMAIN LEUCINE ZIPPER PROTEIN (HD-ZIP) Family HD-Zip proteins are unique to plants, and contain a homeodomain closely linked to a leucine zipper motif involved in dimerization and DNA binding (Ariel et al., 2007). HD-Zip proteins are known to play crucial roles in plant development. The genes in HD-Zip family show variable roles at the different floret/spikelet development, and wheat HD-Zip genes are no exception. Transcript levels for some genes (e.g. TaHDZ-A1, TaHDZ-B10, TaHDZipl-D4) increased from low levels at the WA stage to reach their peak at TP/HD stages (Supplemental Data Set 4). Other genes (e.g. TaHDZB22, TaHDZipl-D4) saw a decrease in their expression level from WA, to reach their lowest level during late stages (Supplemental Data Set 4). ppd-1 mutant alleles lowered the expression of TaHDZipl-A3, TaHDZipl-A4, TaHDZipl-D4, TaHDZ-B10 and TaHDZ-A19 during the AN stage, but raised expression levels of TaHDZ-A9 and TaHDZ-B16 at the same time. The GRAIN NUMBER INCREASE 1 (GNI1) gene encodes an HD-Zip I transcription factor. The mutant GNI-A1 gene is responsible for increased floret fertility in wheat (Sakuma et al., 2019). In our dataset, wild type GNI-A1 expression clearly decreased from WA to YA (Supplemental Data Set 4), which is consistent with previous work (Sakuma et al., 2019). Within individual spikelet, wild type GNI-A1 expression was mainly evident in the most apical Floret4-N position (Supplemental Data Set 5). It indicates the critical role in the determination of floret abortion beyond F3.

EPIDERMAL PATTERNING FACTOR-LIKE (EPFL) Family Secreted peptides mediate intercellular communication (Tavormina et al., 2015). Several secreted peptides in the EPIDERMAL PATTERNING FACTOR-LIKE (EPFL) family regulate plant growth and development, including inflorescence and floret growth and development. EPFL2 and EPFL3 promote shoot growth and organ elongation through the regulation of plant hormones (Kosentka et al., 2019; Tameshige et al., 2016; Uchida et al., 2012). We identified a new EPFL gene and named it TaEPFL-B5 (Supplemental Data Set 4). This new EPFL gene reached its highest transcript levels at the GA stage and its lowest transcript levels at the HD stage.

Genetic analysis in Arabidopsis demonstrated that four putative ligands (EPFL1, EPFL2, EPFL4, and EPFL6) function redundantly in the shoot apical meristem (SAM) by promoting organ elongation (Abrash et al., 2011; Uchida et al., 2012). The wheat gene TaEPFL1 is required for wheat stamen development (Sun et al., 2019). In our dataset, TaEPFL1 expression was high at the GA/YA stage (Supplemental Data Set 4), which is the main stage during which floral organ identity is established. We also saw that the expression of other wheat EPFL genes (TaEPFL-D2, TaEPFL-B3, TaEPFL-A4, TaEPFL5 and TaEPFL-B5) all decreased between the WA and AN stages (Supplemental Data Set 4), suggesting that their roles may be restricted to the early stages of wheat spikelet development. $p p d-1$ mutant alleles lowered transcript levels for TaEPFL-D2, TaEPFL-B3, TaEPFL-A4, TaEPFL5 and TaEPFL-B5 at the AN stage.

Additional Genes Related to Spike Development In maize (Zea mays), ramosa1 (ra1) encodes a $\mathrm{C} 2 \mathrm{H} 2$ zinc-finger transcription factor, while ra3 encodes a trehalose-phosphate phosphatase (Vollbrecht et al., 2005). Both RA1 and RA3 proteins colocalize in a narrow arc of cells at the base of the spikelet-pair meristem. ra3 mutants reduce meristem determinacy and increase tassel branching (Eveland et al., 2014; Satoh-Nagasawa et al., 2006). The histone H2A variant H2A.Z is inserted into chromatin through the highly conserved SWR1 complex (Mizuguchi et al., 2004). Disrupting the activity of SWR1 by silencing one of its key conserved components, ACTIN RELATED PROTEIN 6 (ARP6), triggers floret abortion in Brachypodium 
distachyon (Boden et al., 2013). The expression of the wheat genes TaARP-D6, Tara-B1-like and Tara-A3 decreased from early stages (WA, GA) to the AN stage in our dataset (Supplemental Data Set 4), indicating that any role will be specific to early floret/spikelet development.

The rice transcription factor IDEAL PLANT ARCHITECTURE 1 (IPA1) reduces unproductive tillers and increases grains per panicle, which results in improved yield (Wang et al., 2018). We detected a clear raise in TaIPA-A1 transcript levels between the WA and AN stages (Supplemental Data Set 4), suggesting a role in late floret/spikelet development.

In summary, we can distinguish sets of genes based on their peaks of expression differences along spikelet and floret developmental stages (Figure 5D). Genes for spikelet development with early peaks (WA and GA stages) include TaMADS-D28, TaEPFL-B5, WPI-A1, TaAGL-D17, and TaHDZ-B8. The next wave of genes (TaSOC-A1, TaSVP-B1, TaHDZ-A1, TaAGL$D 8, T a E P F L 1-6 D$, and TaMSD1-2B) reached their peak in transcript levels during the middle of the spike booting stages (YA, TP). Finally, late genes (Tara-D1, Tara-A3, WPI-B2, FUL-D3, TaHDZipl-D4, TaHDZipl-A3, TaHD-A9, TaHDZ-B16, TaHDZ-B10, TaSEP-B1, TaSVP-D3 and TaIPAA1) were maximally detected during late floret/spikelet developmental stages (HD, $A N)$ (Figure 5D).

Genes for floret development with peaks of expression differences at floret $\mathrm{F} 1$ at different stages include TaHDZ-A1(F1-TP, F1-HD), TaSEP-B1 (F1-AN), TaHDZ-A1 (F1-TP, F1-HD), TaEPFL-A2 (F1-TP, F1-HD), and TaSOC-A1 (F1-TP). The next wave of genes (FUL-D3 (F2-TP, F2HD), TaAGL-D17 (F2-TP), TaSVP-B1 (F2-TP), TaSVP-D3 (F2-AN), TaSOC-A1 (F2-HD), Tara-D1 (F2TP, F2-HD), TaHDZ-B10 (F2-HD), and Tara-B3 (F2-HD) reached their peaks in transcript levels at floret F2. The third wave of genes (TaAGL-D17 (F3-HD), TaEPFL-B10 (F3-AN), TaAGL-D17 (F3-HD) reached their peaks of expression differences at floret F3. Finally, the largest differences of gene expression of TaMADS-D28 (F4-TP, F4-HD) and TaEPFL-B10 (F4-TP) were detected at floret F4 (Supplemental Figure 2 and Data Set 5). The results suggest that $P p d-1$ loci may regulate spike architecture through their effects on the regulation of floret development, which is based on the effects of the gene expression related to floret development. 

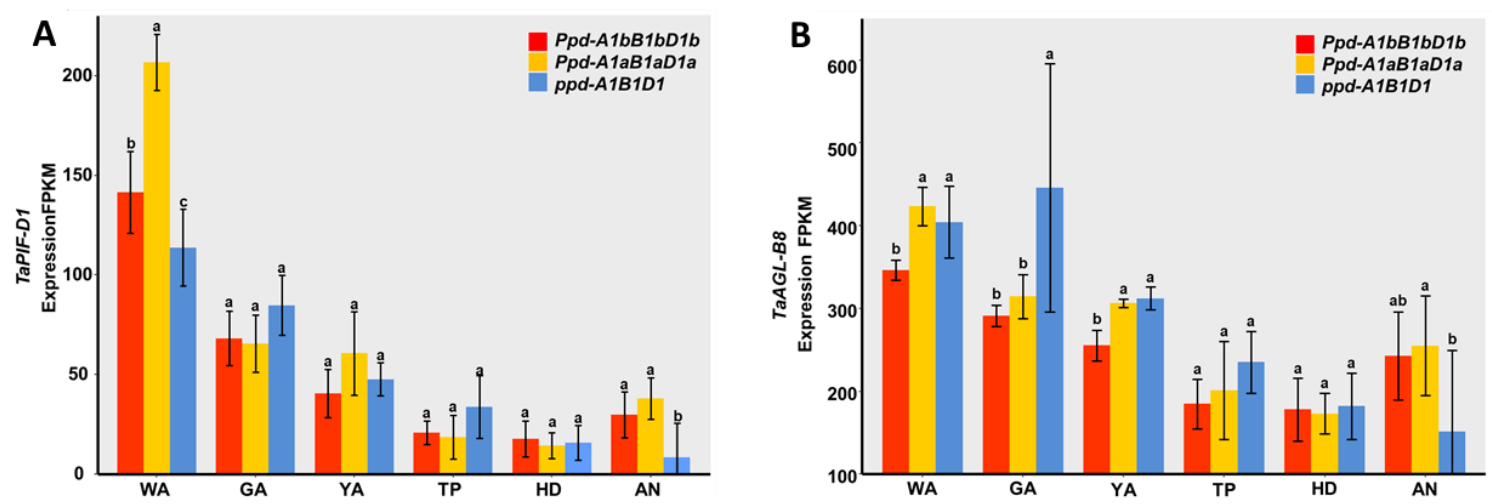

C
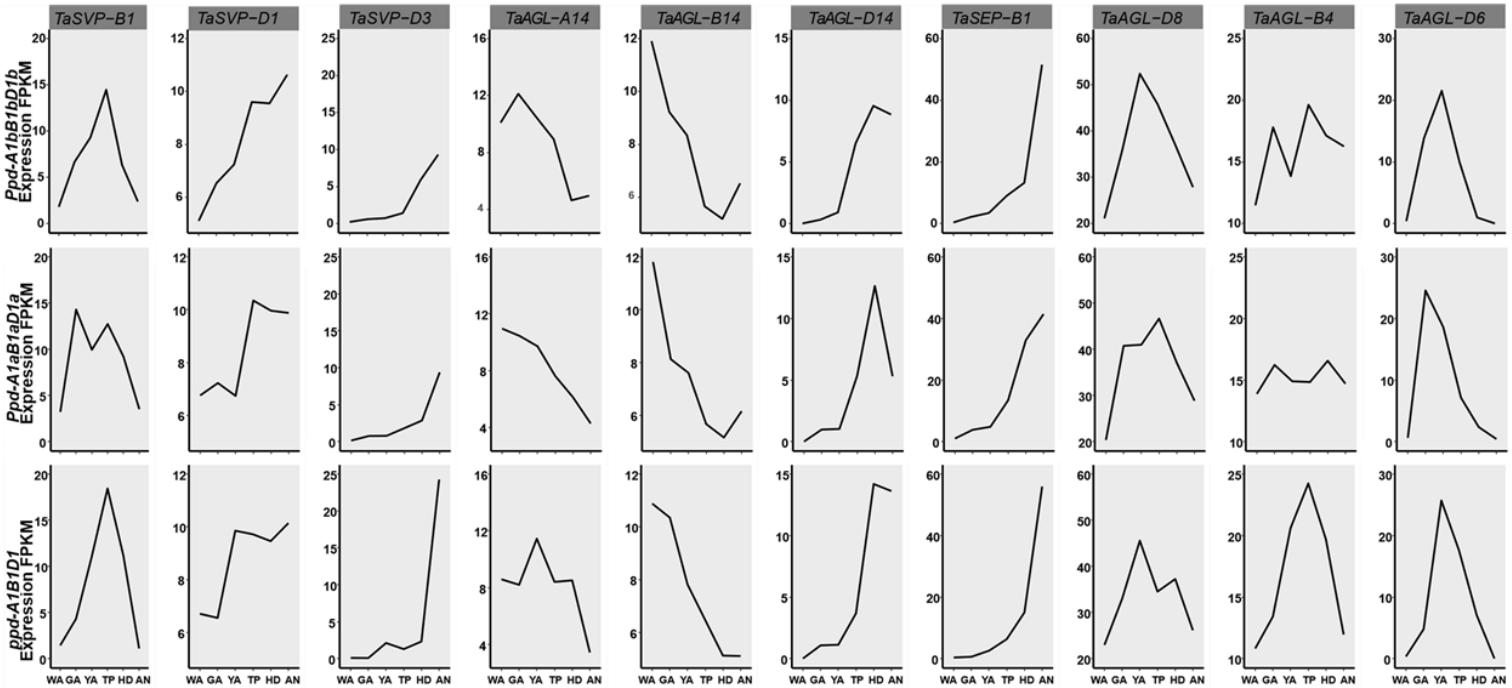

D
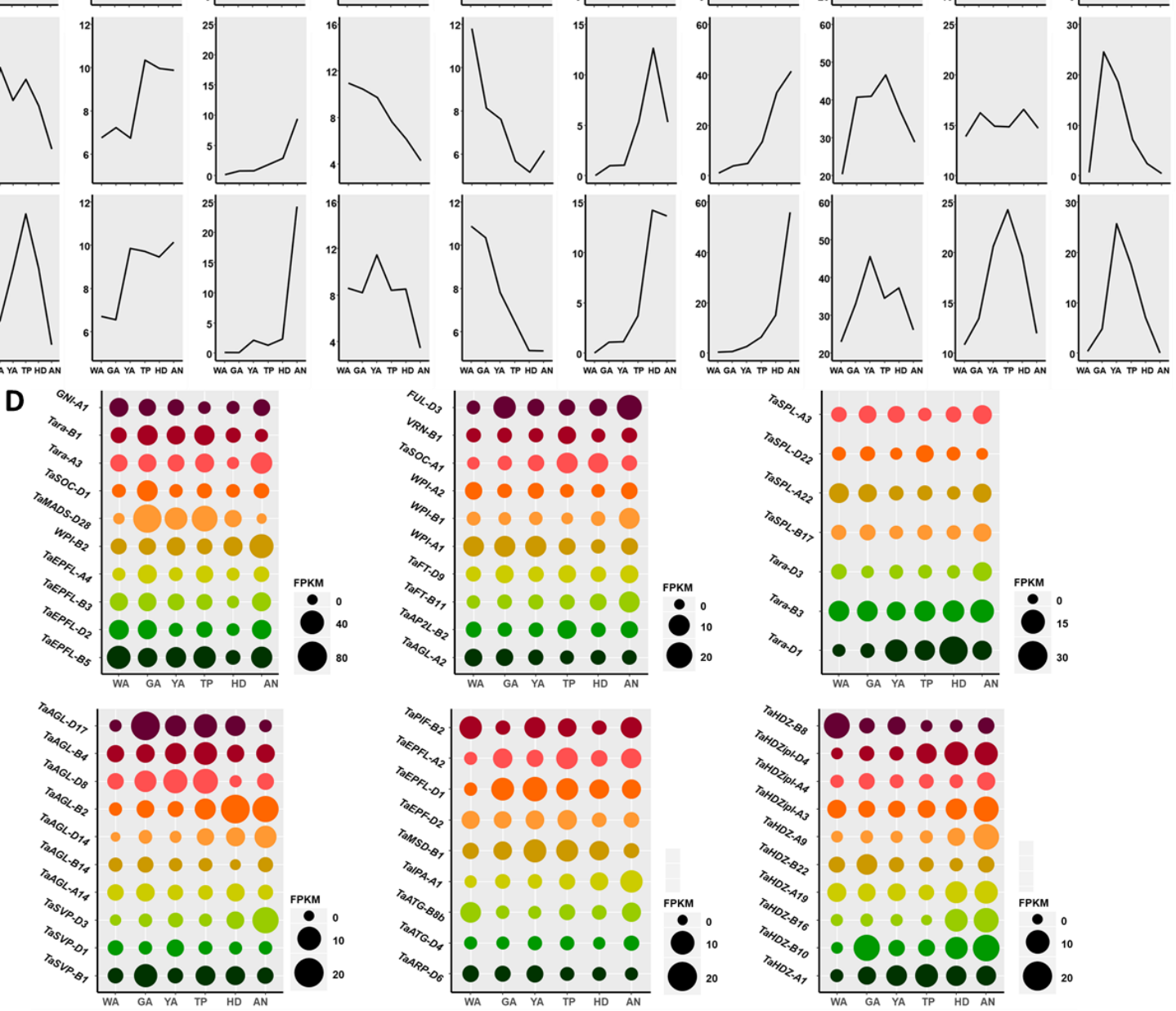

Figure 5. The expression dynamics of MADS-box family genes, SQUAMOSA PROMOTOR BINDING PROTEIN-LIKE (SPL) family, EPIDERMAL PATTERNING FACTOR-LIKE (EPFL) family, PHYTOCHROME INTERACTING FACTOR (PIF) family, Homeodomain leucine zipper protein (HD-ZIP) family and other genes regulating spikelet development.

(A-B) the expression dynamics for the two genes (TaPIF-D1, TaAGL-B8) with highest expression levels.

(C) the expression dynamics of genes in the three $P p d-1$ genotypes $(P p d-A 1 b B 1 b D 1 b, P p d$ $A 1 a B 1 a D 1 a$, and $p p d-A 1 B 1 D 1)$. 
(D) the differences between the highest and lowest expression levels between the three $P p d$ 1 genotypes (Ppd-A1bB1bD1b, Ppd-A1aB1aD1a, and ppd-A1B1D1).

\section{Discussion}

One aim of our study was to determine the effects of various Ppd-1 alleles on spike architecture and development, with the goal to identify new potential strategies for higher yield in wheat cultivars (Figure 6). We also catalogued the effects of Ppd-1 alleles on gene expression patterns during spike, spikelet and floret development, which all contribute to spike architecture in wheat (Figure 6).

We can summarize our study with five main conclusions: First, modulating the duration of spikelet primordia initiation did not always affect spikelet number. Near-Isogenic lines (NILs) carrying photoperiod-insensitive alleles at all three $P p d-1$ loci accelerated flowering time and thus decreased time to terminal spikelet and reduced spikelet number. However, delaying the time necessary to reach the terminal spikelet stage, as in ppd-1 mutant lines did not significantly influence spikelet number. Previous work had shown that FT1, FT2, and Earliness per se (Eps) genes may increase spikelet number by extending the duration of spikelet primordia initiation (Lewis et al., 2008; Finnegan et al., 2018; Shaw et al., 2019). Here, our work suggests that increasing the duration of spikelet primordia initiation with loss of function $p p d-A 1 B 1 D 1$ alleles will not necessarily improve spikelet number. We conclude that $P p d-1$ loci have an intrinsic effect on spikelet number, which regulate the spikelet initiation. In other words, although ppd-A1B1D1 genotypes may slow the rate of spikelet primordia, this effect is counter-balanced by an extension of spikelet primordia formation. Consistent with this hypothesis, Ppd-1, Eps and FT1 genes were reported to influence spikelet number by altering the rate of spikelet initiation (Prieto et al., 2019; Dixon et al., 2018; Ochagavía et al., 2018). Further work will be needed to investigate the mechanism of the spikelet initiation in more details.

A second conclusion of our work is that extending the stem elongation phase (here by the use of the loss of function ppd-A1 B1 D1 alleles) resulted in an improvement of spike chaff components (e.g. rachis, glume, lemma, palea). Again, the converse does not hold: the reduction in the stem elongation phase induced by NILs and their photoperiod-insensitive alleles (Ppd-A1aB1aD1a) did not lead to more floret organs (lemma, palea) at different positions, most likely due to the presence of other floral inhibitor genes e.g. GNI-A1 (Sakuma et al., 2019). Ppd-1 genes greatly affect the rate and duration of floret development (PérezGianmarco et al., 2019; González et al., 2005), which may further regulate floret organs (e.g. lemma, palea). The vascular system within the rachis is critical for grain setting in wheat (Hanif and Langer, 1972). However, information about the effects of $P p d-1$ on rachis is scarce. We observed a marked effect of $P p d-1$ genes on rachis dry weight (Table 4). Any influence on the rachis vascular system may provide a potential explanation for the effects of $P p d-1$ on fertile florets and grain number by modulating the influx of assimilates into developing grains (Wolde and Schnurbusch, 2019).

A third conclusion stems from the observation that extending the pre-anthesis phase (as with the $p p d-1$ triple mutants $p p d-A 1 B 1 D 1$ ) paradoxically reduced phase duration of grain filling post-anthesis resulting in shrivelled grains. Similarly, extending the stem elongation phase caused carpel width increases that were reversed by a shortening of the duration of grain filling. These results indicate the existence of a fine balance between flowering time and grain number: prolonging flowering time may increase fertile floret number/grain number, but over-extension of the flowering window may reduce the duration of grain filling and thus 
negatively influence grain yield. Attempts to raise yield by altering flowering time alone will therefore need to be tread carefully.

Fourth, although most traits such as carpel width were influenced by the genotypes at the Ppd-1 loci, anther length was relatively stable in NILs (Ppd-A1aB1aD1a). Likewise, carpel width responded more strongly to a lengthening of floret development duration (from TS to AN stages) than it did to an acceleration of this developmental transition. Of interest is our observations documenting that Ppd-1 greatly influenced the expression pattern of some genes, which may provide a potential explanation and future avenue of exploration.

Finally, NILs (Ppd-A1aB1aD1a) triggered floral degeneration. The allelic status of the Ppd-1 loci clearly control the rate of spikelet/floret primordia development (Prieto et al., 2018; Ochagavía et al., 2018), further supporting an intrinsic role for Ppd-1 in spike development. The intrinsic effect of $P p d-1$ may also explain its effect on floral degeneration.

These five findings highlight connections between spike architecture and floret developmental traits. We further displayed a high-resolution temporal landscape of the wheat spike transcriptome that provides new insights into floret primordia initiation, development and abortion, illustrating the value of these data sets for investigating diverse aspects of floral development and degeneration. The variable effects of $P p d-1$ alleles on the expression pattern of genes that belong to different families (PIFs, MADS-box, SPL, EPFL and HD-ZIP) at different time windows provide potential explanations for their effects on floret development, and thus spike architecture (Figure 6).

Taken together, our results have revealed connections between spike architecture and floret developmental traits by measuring 51 phenotypic traits in 197 wheat accessions and three Ppd-1 genotypes. Our data set will facilitate the development of future hypotheses and will fuel research into the determinants of floret primordia initiation, development and abortion as well as spike architecture in wheat. Our results suggest that Ppd-1 can remodel spike architecture by regulating floral development in wheat.
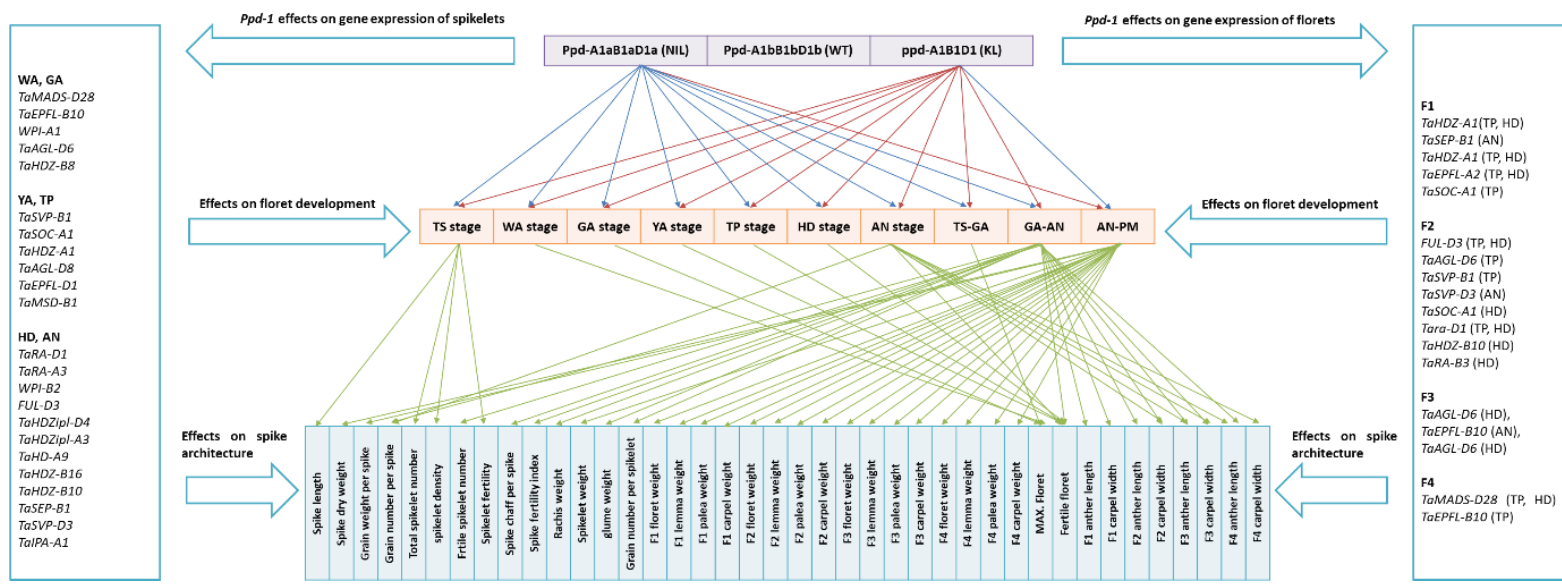

Figure 6. Connections between spike architecture and floral development traits. The blue lines and red lines indicate positive and negative effects, respectively. The green lines suggest the close connections between spike architecture and floret development traits. 


\section{Methods}

\section{Plant Materials and Tissue Preparation}

We grew hexaploid wild type (WT) wheat (Triticum aestivum L. cv. Paragon, a spring wheat carrying photoperiod-sensitive alleles in all three genomes: the triple sensitive allele combination Ppd-A1bB1bD1b), as well as plants from two near-isogenic lines (NILs, triple insensitive allele combination Ppd-A1aB1aD1a) and two independent mutant lines (KLs, triple knockout, $p p d-A 1 B 1 D 1)$ in a greenhouse for phenotypic measurements and transcriptome analysis. Insensitive alleles were derived from the genotypes GS-100 ( $P p d-A 1 a)$, Chinese Spring (CS, $P p d-B 1 a)$, and Sonora 64 ( $P p d-B 1 a$ and $P p d-D 1 a)$. Mutant alleles originated from the genotypes Paragon (Nor ppd-A1 null), Paragon (211a ppd-B1 null), Paragon (319c ppd-B1 null) and Paragon (Nor ppd-D1 null). In more detail, the two NILs are: NIL1 (GS-100 2A + CS 2B + Sonora 64 2D), NIL2 (GS-100 2A + Sonora 64 2B + Sonora 64 2D). The two mutant lines are: KL1 (Nor ppd-A1 null + 211a ppd-B1 null + Nor ppd-D1 null), KL2 (Nor ppd-A1 null + 319c ppd$B 1$ null + Nor ppd-D1 null). The generation of NILs and mutant lines was described previously (Shaw et al., 2013; Bentley et al., 2013). We used all 5 genotypes for phenotypic characterization, and 3 genotypes for transcriptome analysis: WT, NIL2 and KL2.

We selected 197 European hexaploid winter wheat cultivars from a previous genomewide association study (210 cultivars) (Guo et al., 2017b). We genotyped photoperiodsensitivity and photoperiod-insensitivity of photoperiod (Ppd-D1) (chromosome 2D) in this 197 cultivars. Of the 197 wheat cultivars, 165 cultivars are photoperiod-sensitive and 32 cultivars are photoperiod-in sensitive. Finally, we determined the effects of photoperiodsensitivity and photoperiod-insensitivity of photoperiod (Ppd-D1) on the phenotypes (Guo et al., 2017b, 2018).

We sowed grains in 96-well trays and germinated them under greenhouse conditions (photoperiod, $16 \mathrm{~h} / 8 \mathrm{~h}$, light/dark; temperature, $20^{\circ} \mathrm{C} / 16^{\circ} \mathrm{C}$ ) for $14 \mathrm{~d}$. We then vernalized seedlings at $4^{\circ} \mathrm{C}$ for $28 \mathrm{~d}$. following which we transferred them to $15^{\circ} \mathrm{C}$ for one week to gradually acclimatize. Finally, we transplanted seedlings into pots, one per pot $(9 \times 9 \times 9$ $\mathrm{cm})$. Supplemental light was provided and plants were irrigated when required.

We conducted temporal-spatial dissection to obtain overall information on floret development and abortion at six floral developmental stages of wheat: the white anther (WA) stage, green anther (GA) stage (maximum number of floret primordium), yellow anther (YA) stage, tipping (TP) stage, heading (HD) stage and anthesis (AN) stage. Details about the determination of different developmental stages can be found in (Guo et al., 2016; Guo and Schnurbusch, 2015). At each stage, the main shoots of three plants from each genotype were randomly selected to determine the number of floret primordia per spikelet. We took the entire spikelet (without the glume, from the spikelets in the centre of the spike) at each stage for temporal transcriptome analysis of spikelet (Figure 2). We took individual florets from four positions (F1, F2, F3 and F4-N, Figure 2) within individual spikelets (from spikelets in the centre of the spike) for temporal-spatial transcriptome analysis of floret (Figure 2). We collected four to five biological replicates for each time point, each replicate being a pool from at least three to ten plants. Total RNA was extracted using TRIzol reagent (Invitrogen).

\section{Read Mapping and Transcript Profiling}

We downloaded the Chinese Spring reference genome from https://urgi.versailles.inra.fr/download/iwgsc/IWGSC RefSeq Assemblies/v1.0/. We assessed the quality of raw data using Fastqc (http://www.bioinformatics.babraham.ac.uk/projects/fastqc/). We then processed raw RNA- 
seq reads by first removing low-quality reads using trimmomatic (Bolger et al., 2014) and then mapped trimmed reads to the Chinese Spring reference genome using Hisat2 (Kim et al., 2015). After alignment, we normalized raw counts for each wheat gene to fragments per kilobase million mapped reads (FPKM). Gene expression counts were tabulated with HTseq-count (Anders et al., 2015). We determined significant differences in gene expression based on FPKM counts with the DESeq2 (Love et al., 2014) package in R. If the expression of one gene is below 1 , we considered the gene was not expressed, which was further used to determine the stagespecific and position-specific genes.

\section{Gene Annotation and Functional Enrichment Analysis}

We obtained gene models and annotations from the International Wheat Genome Sequencing Consortium database

(https://urgi.versailles.inra.fr/download/iwgsc/IWGSC RefSeq Annotations/v1.1/). We obtained Gene Ontology (GO) terms for wheat genes from the ensembl plant database (http://plants.ensembl.org/index.html). We performed GO enrichment analysis with AgriGO (Du et al., 2010) and applied a hypergeometric test with rich factor correction (adjusted $P$ value $<0.05)$.

\section{Transmission Electron Microscopy}

For comparative ultrastructural analysis, wheat spikelets at different developmental stages were used for combined conventional and microwave-assisted tissue preparation. Aldehyde fixation, substitution and resin embedding were performed as defined in Supplemental Protocol (Supplemental Table 1). Sectioning and ultrastructure analysis were performed as described previously (Daghma et al., 2011).

\section{Acknowledgements}

We thank Dr Simon Griffiths and the Germplasm Resources Unit at John Innes Centre (Norwich, UK) for providing grains of the Ppd-1 genotypes. Marion Roeder (Leibniz Institute of Plant Genetics and Crop Plant Research) and Martin Ganal (Trait Genetics GmbH) provided the 197 wheat accessions and the information of Ppd-D1 allele in these accessions. We thank Dr. Martin Mascher (Leibniz Institute of Plant Genetics and Crop Plant Research), Yingyin Yao (China Agricultural University) and Jun Xiao (Institute of Genetics and Developmental Biology, Chinese Academy of Sciences) for suggestions about writing and data analysis. We thank Kirsten Hoffie and Marion Benecke for technical support in transmission electron microscopy. This work was supported by the Strategic Priority Research Program of Chinese Academy of Sciences, Grant No. XDA/B 00000000. Research in the Schnurbusch lab received financial support from the HEISENBERG Program of the German Research Foundation (DFG), grant no. SCHN 768/8-1 and the IPK core budget.

\section{Author contributions}

Z.G. and T.S. designed and supervised the experiments. Z.G. performed the experiments and wrote the manuscript. Y.L. and L.Z. performed data analyses and prepared figures. M.M. conducted the analysis of cellular morphology. All authors viewed and edited the manuscript.

\section{Competing interests}

The authors declare no competing financial interests. 


\section{Supplemental Table 1. Protocol for combined conventional and microwave-assisted fixation, dehydration and Spurr resin embedding of wheat spikelets for ultrastructural analysis.}

\begin{tabular}{|c|c|c|c|c|}
\hline \multicolumn{5}{|c|}{$\begin{array}{l}\text { Combined conventional and microwave-assisted tissue preparation in a } \\
\text { PELCO Bio Wave }{ }^{\circledR} 34700-230 \text { (Ted Pella, Inc., Redding, CA, USA) }\end{array}$} \\
\hline Process & Reagent & $\begin{array}{l}\text { Power } \\
\text { [W] }\end{array}$ & $\begin{array}{l}\text { Time } \\
\text { [sec] }\end{array}$ & $\begin{array}{l}\text { Vacuum } \\
\text { [mm Hg] }\end{array}$ \\
\hline 1. Primary fixation & $\begin{array}{l}2.0 \%(\mathrm{v} / \mathrm{v}) \text { gluTaraldehyde and } 2.0 \%(\mathrm{v} / \mathrm{v}) \\
\text { paraformaldehyde in } 0.05 \mathrm{M} \text { cacodylate } \\
\text { buffer }(\mathrm{pH} 7.3)\end{array}$ & $\begin{array}{c}0 \\
150 \\
0 \\
150\end{array}$ & $\begin{array}{l}60 \\
60 \\
60 \\
60\end{array}$ & $\begin{array}{l}0 \\
0 \\
0 \\
0\end{array}$ \\
\hline 2. Wash & $\begin{array}{l}1 \times 0.05 \mathrm{M} \text { cacodylate buffer }(\mathrm{pH} 7.3) \\
\text { and } 2 \times \text { aqua dest. }\end{array}$ & 150 & 45 & 0 \\
\hline \multirow[t]{2}{*}{ 3. Secondary fixation } & $1 \%(\mathrm{v} / \mathrm{v})$ osmium tetroxide in aqua dest. & $\begin{array}{c}0 \\
80 \\
0 \\
80\end{array}$ & $\begin{array}{c}60 \\
120 \\
60 \\
120\end{array}$ & $\begin{array}{l}10 \\
10 \\
10 \\
10\end{array}$ \\
\hline & \multicolumn{4}{|c|}{ Keep samples an additional 30 min on a shaker. } \\
\hline 4. Wash & $3 \times$ aqua dest. & 150 & 45 & 0 \\
\hline \multirow[t]{2}{*}{ 5. Dehydration } & $\begin{array}{l}\text { Acetone: } 30 \%, 40 \%, 50 \%, 60 \%, 70 \% \text {, } \\
80 \%, 90 \%, 1 \times 100 \% \text { and } 1 \times \\
\text { propylenoxide. }\end{array}$ & 150 & 45 & 0 \\
\hline & \multicolumn{4}{|c|}{ After each step, keep samples an additional 10 min on a shaker. } \\
\hline 6. Resin infiltration & $\begin{array}{l}25 \% \text { Spurr resin in propylenoxide } \\
50 \% \text { Spurr resin in propylenoxide } \\
75 \% \text { Spurr resin in propylenoxide } \\
100 \% \text { Spurr resin }\end{array}$ & \multicolumn{3}{|c|}{$\begin{array}{l}\text { Overnight on shaker at RT } \\
4 \text { hrs on shaker at RT } \\
4 \text { hrs on shaker at RT } \\
\text { Overnight on shaker at RT }\end{array}$} \\
\hline 7. Polymerization & \multicolumn{4}{|c|}{$24 \mathrm{hrs}$ at $70^{\circ} \mathrm{C}$ in flat embedding moulds in a heating cabinet. } \\
\hline
\end{tabular}



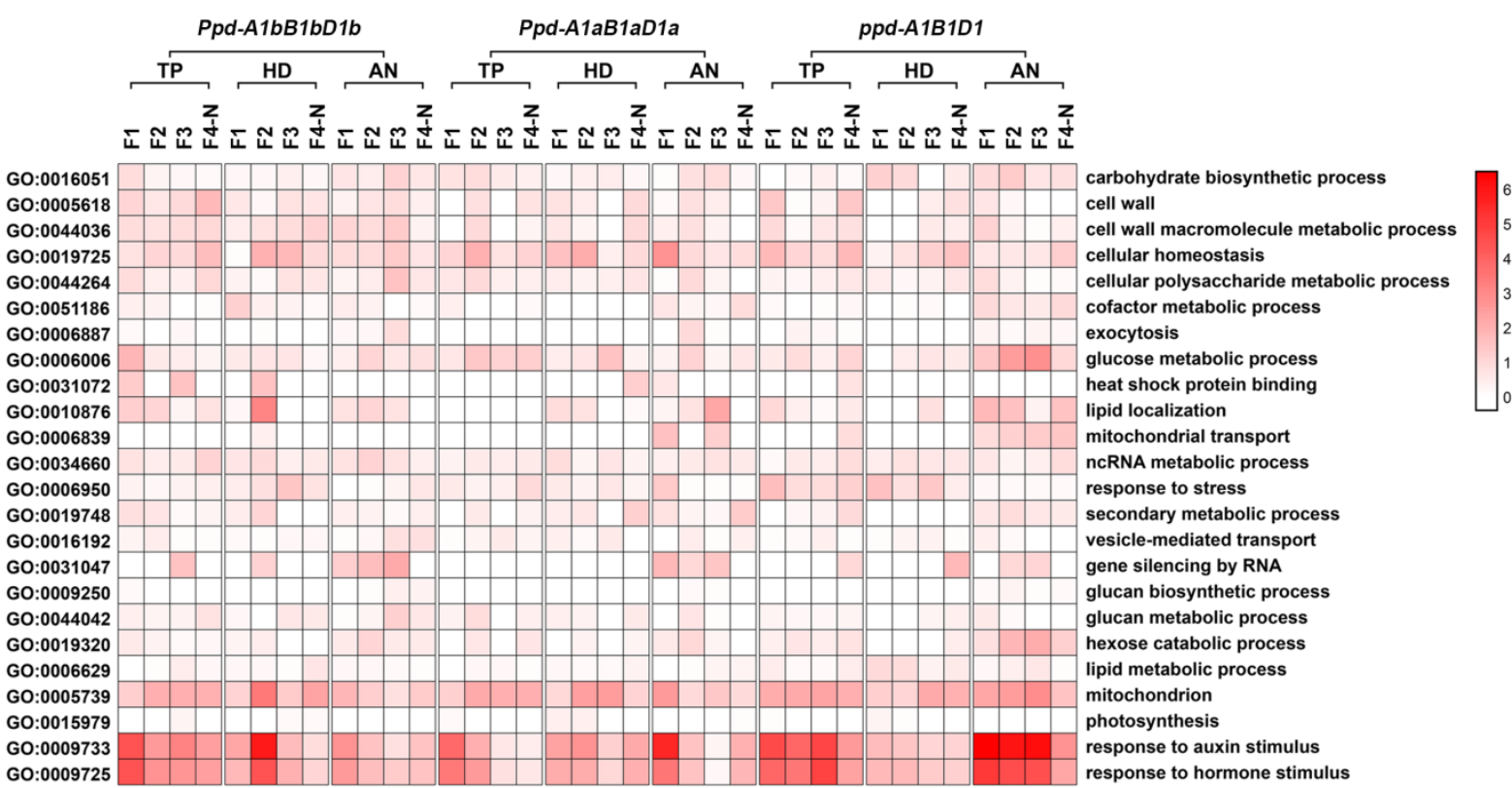

Supplementary Figure 1. Temporal-spatial functional transition of floret genes across three developmental stages and four floret positions in the three Ppd-1 genotypes. The three stages (TP, HD and AN) and four floret positions (F2, F2, F3 and F4-N) are shown in Figure 1 and the three $P p d-1$ genotypes ( $P p d-A 1 b B 1 b D 1 b, P p d-A 1 a B 1 a D 1 a$, and $p p d-A 1 B 1 D 1$ ) in Table 1. The depth of the red colour indicates the dynamic changes of factors across the stages and positions. 

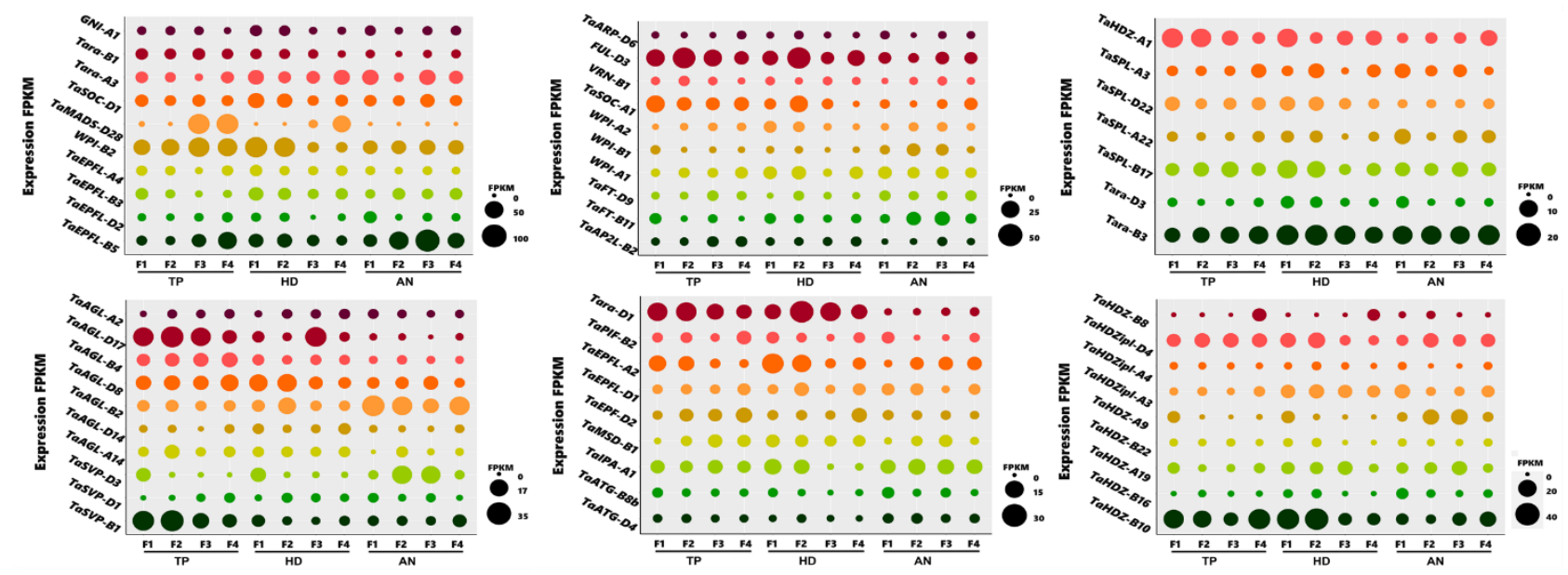

Supplemental Figure 2. The differences between the highest and lowest expression levels between the three Ppd-1 genotypes (Ppd-A1bB1bD1b, Ppd-A1aB1aD1a, and ppd-A1B1D1) for the MADS-box family genes, SQUAMOSA PROMOTOR BINDING PROTEIN-LIKE (SPL) family, EPIDERMAL PATTERNING FACTOR-LIKE (EPFL) family, PHYTOCHROME INTERACTING FACTOR (PIF) family, Homeodomain leucine zipper protein (HD-ZIP) family and other genes regulating floret development. 


\section{Reference}

Abrash, E.B., Davies, K.A., and Bergmann, D.C. (2011). Generation of signaling specificity in Arabidopsis by spatially restricted buffering of ligand-receptor interactions. Plant Cell.

Anders, S., Pyl, P.T., and Huber, W. (2015). HTSeq-A Python framework to work with highthroughput sequencing data. Bioinformatics.

Ariel, F.D., Manavella, P.A., Dezar, C.A., and Chan, R.L. (2007). The true story of the HD-Zip family. Trends Plant Sci.

Avin-Wittenberg, T., Bajdzienko, K., Wittenberg, G., Alseekh, S., Tohge, T., Bock, R., Giavalisco, P., and Fernie, A.R. (2015). Global analysis of the role of autophagy in cellular metabolism and energy homeostasis in arabidopsis seedlings under carbon starvation. Plant Cell.

Balanzà, V., Martínez-Fernández, I., and Ferrándiz, C. (2014). Sequential action of FRUITFULL as a modulator of the activity of the floral regulators SVP and SOC1. J. Exp. Bot.

Beales, J., Turner, A., Griffiths, S., Snape, J.W., and Laurie, D.A. (2007). A Pseudo-Response Regulator is misexpressed in the photoperiod insensitive Ppd-D1a mutant of wheat (Triticum aestivum L.). Theor. Appl. Genet.

Behrends, C., Sowa, M.E., Gygi, S.P., and Harper, J.W. (2010). Network organization of the human autophagy system. Nature.

Bentley, A.R. et al. (2013). Short, natural, and extended photoperiod response in BC2F 4 lines of bread wheat with different Photoperiod-1 (Ppd-1) alleles. J. Exp. Bot.

Béziat, C., Barbez, E., Feraru, M.I., Lucyshyn, D., and Kleine-Vehn, J. (2017). Light triggers PILS-dependent reduction in nuclear auxin signalling for growth transition. Nat. Plants.

Boden, S.A., Cavanagh, C., Cullis, B.R., Ramm, K., Greenwood, J., Jean Finnegan, E., Trevaskis, B., and Swain, S.M. (2015). Ppd-1 is a key regulator of inflorescence architecture and paired spikelet development in wheat. Nat. Plants 1.

Boden, S.A., Kavanová, M., Finnegan, E.J., and Wigge, P.A. (2013). Thermal stress effects on grain yield in Brachypodium distachyon occur via H2A.Z-nucleosomes. Genome Biol.

Bolger, a. M., Lohse, M., and Usadel, B. (2014). Trimmomatic: A flexible read trimming tool for Illumina NGS data. Bioinformatics.

Cheng, H. (2004). Gibberellin regulates Arabidopsis floral development via suppression of DELLA protein function. Development 131: 1055-1064.

Collani, S., Neumann, M., Yant, L., and Schmid, M. (2019). FT modulates genome-wide DNAbinding of the bZIP transcription factor FD. Plant Physiol.

Daghma, D.S., Kumlehn, J., and Melzer, M. (2011). The use of cyanobacteria as filler in nitrocellulose capillaries improves ultrastructural preservation of immature barley pollen upon high pressure freezing. J. Microsc.

Debernardi, J.M., Greenwood, J.R., Jean Finnegan, E., Jernstedt, J., and Dubcovsky, J. (2019). APETALA 2-like genes AP2L2 and Q specify lemma identity and axillary floral meristem development in wheat. Plant J.

Dixon, L.E., Farré, A., Finnegan, E.J., Orford, S., Griffiths, S., and Boden, S.A. (2018). Developmental responses of bread wheat to changes in ambient temperature following deletion of a locus that includes FLOWERING LOCUS T1. Plant Cell Environ.

Du, Z., Zhou, X., Ling, Y., Zhang, Z., and Su, Z. (2010). agriGO: A GO analysis toolkit for the agricultural community. Nucleic Acids Res.

Eveland, A.L. et al. (2014). Regulatory modules controlling maize inflorescence architecture. Genome Res. 
Fan, J., Yu, L., and Xu, C. (2019). Dual Role for Autophagy in Lipid Metabolism in Arabidopsis. Plant Cell.

Figueiredo, D.D., Batista, R.A., Roszak, P.J., and Köhler, C. (2015). Auxin production couples endosperm development to fertilization. Nat. Plants.

Finnegan, E.J. et al. (2018). Zebularine treatment is associated with deletion of FT-B1 leading to an increase in spikelet number in bread wheat. Plant Cell Environ.

Flanagan, C.A. and Ma, H. (1994). Spatially and temporally regulated expression of the MADS-box gene AGL2 in wild-type and mutant arabidopsis flowers. Plant Mol. Biol.

Ghiglione, H.O., Gonzalez, F.G., Serrago, R., Maldonado, S.B., Chilcott, C., Curá, J.A., Miralles, D.J., Zhu, T., and Casal, J.J. (2008). Autophagy regulated by day length determines the number of fertile florets in wheat. Plant J.

González, F.G., Slafer, G.A., and Miralles, D.J. (2005). Pre-anthesis development and number of fertile florets in wheat as affected by photoperiod sensitivity genes Ppd-D1 and Ppd-B1. Euphytica 146: 253-269.

Gu, Q., Ferrándiz, C., Yanofsky, M.F., and Martienssen, R. (1998). The FRUITFULL MADS-box gene mediates cell differentiation during Arabidopsis fruit development. Development.

Guo, Z., Chen, D., Alqudah, A.M., Röder, M.S., Ganal, M.W., and Schnurbusch, T. (2017a). Genome-wide association analyses of 54 traits identified multiple loci for the determination of floret fertility in wheat. New Phytol. 214: 257-270.

Guo, Z., Chen, D., Alqudah, A.M., Röder, M.S., Ganal, M.W., and Schnurbusch, T. (2017b). Genome-wide association analyses of 54 traits identified multiple loci for the determination of floret fertility in wheat. New Phytol. 214: 257-270.

Guo, Z., Chen, D., Röder, M.S., Ganal, M.W., and Schnurbusch, T. (2018). Genetic dissection of pre-anthesis sub-phase durations during the reproductive spike development of wheat. Plant J.

Guo, Z., Chen, D., and Schnurbusch, T. (2015). Variance components, heritability and correlation analysis of anther and ovary size during the floral development of bread wheat. J. Exp. Bot. 66: 3099-3111.

Guo, Z. and Schnurbusch, T. (2015). Variation of floret fertility in hexaploid wheat revealed by tiller removal. J. Exp. Bot. 66: 5945-5958.

Guo, Z., Slafer, G.A., and Schnurbusch, T. (2016). Genotypic variation in spike fertility traits and ovary size as determinants of floret and grain survival rate in wheat. J. Exp. Bot.

Hama, E., Takumi, S., Ogihara, Y., and Murai, K. (2004). Pistillody is caused by alterations to the class-B MADS-box gene expression pattern in alloplasmic wheats. Planta.

Hanif, M. and Langer, R.H.M. (1972). The vascular system of the spikelet in wheat (Triticum aestivum). Ann. Bot.

Hong, Y. and Jackson, S. (2015). Floral induction and flower formation-the role and potential applications of miRNAs. Plant Biotechnol. J.

Jung, J.H., Lee, H.J., Ryu, J.Y., and Park, C.M. (2016). SPL3/4/5 Integrate Developmental Aging and Photoperiodic Signals into the FT-FD Module in Arabidopsis Flowering. Mol. Plant.

Kato, K. and Yokoyama, H. (1992). Geographical variation in heading characters among wheat landraces, Triticum aestivum L., and its implication for their adaptability. Theor. Appl. Genet.

Kim, D., Langmead, B., and Salzberg, S.L. (2015). HISAT: A fast spliced aligner with low memory requirements. Nat. Methods.

Kirby, E. and Appleyard, M. (1987). Cereal development guide. 2nd Edition. 2nd ed. (NAC Cereal Unit.: Stoneleigh, UK). 
Kosentka, P.Z., Overholt, A., Maradiaga, R., Mitoubsi, O., and Shpak, E.D. (2019). EPFL signals in the boundary region of the SAM restrict its size and promote leaf initiation. Plant Physiol.

Krizek, B.A. and Fletcher, J.C. (2005). Molecular mechanisms of flower development: An armchair guide. Nat. Rev. Genet.

Lee, J.H., Kim, J.J., and Ahn, J.H. (2012). Role of SEPALLATA3 (SEP3) as a downstream gene of miR156-SPL3-FT circuitry in ambient temperature-responsive flowering. Plant Signal. Behav.

Lewis, S., Faricelli, M.E., Appendino, M.L., Val??rik, M., and Dubcovsky, J. (2008). The chromosome region including the earliness per se locus Eps-A $\mathrm{m} 1$ affects the duration of early developmental phases and spikelet number in diploid wheat. J. Exp. Bot. 59: 3595-3607.

Li, C., Lin, H., Chen, A., Lau, M., Jernstedt, J., and Dubcovsky, J. (2019). Wheat VRN1 , FUL2 and FUL3 play critical and redundant roles in spikelet development and spike determinacy. Development.

Li, D. et al. (2016). FAR-RED ELONGATED HYPOCOTYL3 activates SEPALLATA2 but inhibits CLAVATA3 to regulate meristem determinacy and maintenance in Arabidopsis. Proc. Natl. Acad. Sci. U. S. A.

Love, M.I., Huber, W., and Anders, S. (2014). Moderated estimation of fold change and dispersion for RNA-seq data with DESeq2. Genome Biol.

Ma, H., Yanofsky, M.F., and Meyerowitz, E.M. (1991). AGL1-AGL6, an Arabidopsis gene family with similarity to floral homeotic and transcription factor genes. Genes Dev.

Mandel, M.A. and Yanofsky, M.F. (1995). The Arabidopsis AGL8 MADS box gene is expressed in inflorescence meristems and is negatively regulated by APETALA1. Plant Cell.

McIntosh, R.A., Yamazaki, Y., Devos, K.M., Dubcovsky, J., Rogers, W.J., and Appels, R. (2003). Catalogue of Gene Symbols for Wheat. 10th Int. Wheat Genet. Symp.

Mizuguchi, G., Shen, X., Landry, J., Wu, W.H., Sen, S., and Wu, C. (2004). ATP-Driven Exchange of Histone H2AZ Variant Catalyzed by SWR1 Chromatin Remodeling Complex. Science (80-. ).

Moore, B., Zhou, L., Rolland, F., Hall, Q., Cheng, W.H., Liu, Y.X., Hwang, I., Jones, T., and Sheen, J. (2003). Role of the Arabidopsis glucose sensor HXK1 in nutrient, light, and hormonal signaling. Science (80-. ).

Murrow, L., Malhotra, R., and Debnath, J. (2015). ATG12-ATG3 interacts with Alix to promote basal autophagic flux and late endosome function. Nat. Cell Biol.

Mutasa-Göttgens, E. and Hedden, P. (2009). Gibberellin as a factor in floral regulatory networks. J. Exp. Bot. 60: 1979-1989.

Ochagavía, H., Prieto, P., Savin, R., Griffiths, S., and Slafer, G. (2018). Dynamics of leaf and spikelet primordia initiation in wheat as affected by Ppd-1a alleles under field conditions. J. Exp. Bot.

Oh, E., Kim, J., Park, E., Kim, J. II, Kang, C., and Choi, G. (2004). PIL5, a phytochromeinteracting basic helix-loop-helix protein, is a key negative regulator of seed germination in Arabidopsis thaliana. Plant Cell.

Ohmori, S., Kimizu, M., Sugita, M., Miyao, A., Hirochika, H., Uchida, E., Nagato, Y., and Yoshida, H. (2009). MOSAIC FLORAL ORGANS1, an AGL6-like MADS box gene, regulates floral organ identity and meristem fate in rice. Plant Cell.

Pérez-Gianmarco, T.I., Slafer, G.A., and González, F.G. (2019). Photoperiod-sensitivity genes shape floret development in wheat. J. Exp. Bot. 
Pérez-Ruiz, R. V. et al. (2015). XAANTAL2 (AGL14) is an important component of the complex gene regulatory network that underlies arabidopsis shoot apical meristem transitions. Mol. Plant.

Pham, V.N., Kathare, P.K., and Huq, E. (2018). Phytochromes and phytochrome interacting factors. Plant Physiol.

Prasad, K. and Vijayraghavan, U. (2003). Double-Stranded RNA Interference of a Rice PI/GLO Paralog, OsMADS2, Uncovers Its Second-Whorl-Specific Function in Floral Organ Patterning. Genetics.

Prieto, P., Ochagavía, H., Griffiths, S., and Slafer, G.A. (2019). Earliness per sextemperature interaction: consequences on leaf, spikelet, and floret development in wheat. J. Exp. Bot.

Prieto, P., Ochagavía, H., Savin, R., Griffiths, S., and Slafer, G.A. (2018). Dynamics of floret initiation/death determining spike fertility in wheat as affected by Ppd genes under field conditions. J. Exp. Bot.

Sakuma, S. et al. (2019). Unleashing floret fertility in wheat through the mutation of a homeobox gene. Proc. Natl. Acad. Sci. U. S. A.

Satoh-Nagasawa, N., Nagasawa, N., Malcomber, S., Sakai, H., and Jackson, D. (2006). A trehalose metabolic enzyme controls inflorescence architecture in maize. Nature.

Scarth, R. and Law, C.N. (1983). The location of the photoperiod gene, Ppd2 and an additional genetic factor for ear-emergence time on chromosome $2 \mathrm{~B}$ of wheat. Heredity (Edinb).

Schauer, S.E., Schlüter, P.M., Baskar, R., Gheyselinck, J., Bolaños, A., Curtis, M.D., and Grossniklaus, U. (2009). Intronic regulatory elements determine the divergent expression patterns of agamous-like6 subfamily members in arabidopsis. Plant J.

Shaw, L.M., Lyu, B., Turner, R., Li, C., Chen, F., Han, X., Fu, D., and Dubcovsky, J. (2019). FLOWERING LOCUS T2 regulates spike development and fertility in temperate cereals. J. Exp. Bot.

Shaw, L.M., Turner, A.S., Herry, L., Griffiths, S., and Laurie, D.A. (2013). Mutant alleles of Photoperiod-1 in Wheat (Triticum aestivum L.) that confer a late flowering phenotype in long days. PLoS One.

Shaw, L.M., Turner, A.S., and Laurie, D.A. (2012). The impact of photoperiod insensitive Ppd-1a mutations on the photoperiod pathway across the three genomes of hexaploid wheat (Triticum aestivum). Plant J. 71: 71-84.

Song, S., Chen, Y., Liu, L., See, Y.H.B., Mao, C., Gan, Y., and Yu, H. (2018). OsFTIP7 determines auxin-mediated anther dehiscence in rice. Nat. Plants.

Sun, Q., Qu, J., Yu, Y., Yang, Z., Wei, S., Wu, Y., Yang, J., and Peng, Z. (2019). TaEPFL1, an EPIDERMAL PATTERNING FACTOR-LIKE (EPFL) secreted peptide gene, is required for stamen development in wheat. Genetica.

Tameshige, T., Okamoto, S., Lee, J.S., Aida, M., Tasaka, M., Torii, K.U., and Uchida, N. (2016). A Secreted Peptide and Its Receptors Shape the Auxin Response Pattern and Leaf Margin Morphogenesis. Curr. Biol.

Tavormina, P., De Coninck, B., Nikonorova, N., De Smet, I., and Cammuea, B.P.A. (2015). The plant peptidome: An expanding repertoire of structural features and biological functions. Plant Cell.

Thompson, B.E., Bartling, L., Whipple, C., Hall, D.H., Sakai, H., Schmidt, R., and Hake, S. (2009). Bearded-ear encodes a MADS box transcription factor critical for maize floral development. Plant Cell. 
Uchida, N., Lee, J.S., Horst, R.J., Lai, H.H., Kajita, R., Kakimoto, T., Tasaka, M., and Torii, K.U. (2012). Regulation of inflorescence architecture by intertissue layer ligand-receptor communication between endodermis and phloem. Proc. Natl. Acad. Sci. U. S. A.

Vollbrecht, E., Springer, P.S., Goh, L., Buckler IV, E.S., and Martienssen, R. (2005). Architecture of floral branch systems in maize and related grasses. Nature.

Wang, J. et al. (2018). A single transcription factor promotes both yield and immunity in rice. Science (80-. ).

Wigge, P.A. (2011). FT, A mobile developmental signal in plants. Curr. Biol.

Wigge, P.A., Kim, M.C., Jaeger, K.E., Busch, W., Schmid, M., Lohmann, J.U., and Weigel, D. (2005). Integration of spatial and temporal information during floral induction in Arabidopsis. Science (80-. ).

Wilhelm, E.P., Turner, A.S., and Laurie, D.A. (2009). Photoperiod insensitive Ppd-A1a mutations in tetraploid wheat (Triticum durum Desf.). Theor. Appl. Genet. 118: 285294.

Wolde, G.M. and Schnurbusch, T. (2019). Inferring vascular architecture of the wheat spikelet based on resource allocation in the branched headt (bht - A1) near isogenic lines. Funct. Plant Biol.

Worland, A.J., Börner, A., Korzun, V., Li, W.M., Petrovíc, S., and Sayers, E.J. (1998). The influence of photoperiod genes on the adaptability of European winter wheats. In Euphytica.

Wu, M., Liu, D., Abdul, W., Upreti, S., Liu, Y., Song, G., Wu, J., Liu, B., and Gan, Y. (2018). PIL5 represses floral transition in Arabidopsis under long day conditions. Biochem. Biophys. Res. Commun.

Xia, L., Bin, Z., Xinguo, M., Ang, L., Meirong, S., and Ruilian, J. (2014). [Cloning of taeMIR156 precursor gene and sequence polymorphisms of tae-miR156 targeted TaSPL17]. Yi Chuan.

Yadav, S.R., Prasad, K., and Vijayraghavan, U. (2007). Divergent regulatory OsMADS2 functions control size, shape and differentiation of the highly derived rice floret secondwhorl organ. Genetics.

Yamaguchi, A., Wu, M.F., Yang, L., Wu, G., Poethig, R.S., and Wagner, D. (2009). The MicroRNA-Regulated SBP-Box Transcription Factor SPL3 Is a Direct Upstream Activator of LEAFY, FRUITFULL, and APETALA1. Dev. Cell.

Yan, L., Loukoianov, A., Tranquilli, G., Helguera, M., Fahima, T., and Dubcovsky, J. (2003). Positional cloning of the wheat vernalization gene VRN1. Proc. Natl. Acad. Sci. U. S. A.

Yu, J., Zhao, W., Tong, W., He, Q., Yoon, M.Y., Li, F.P., Choi, B., Heo, E.B., Kim, K.W., and Park, Y.J. (2018). A genome-wide association study reveals candidate genes related to salt tolerance in rice (Oryza sativa) at the germination stage. Int. J. Mol. Sci.

Zadoks, J.C., Chang, T.T., and Konzak, C.F. (1974). A decimal code for the growth stages of cereals. Weed Res. 14: 415-421. 